\title{
MITEs in the promoters of effector genes allow prediction of novel virulence genes in Fusarium oxysporum
}

\author{
Sarah M Schmidt ${ }^{1}$, Petra M Houterman ${ }^{1}$, Ines Schreiver ${ }^{1,4}$, Lisong Ma ${ }^{1}$ Stefan Amyotte ${ }^{2}$, Biju Chellappan , \\ Sjef Boeren ${ }^{3}$, Frank L W Takken ${ }^{1}$ and Martijn Rep ${ }^{1 *}$
}

\begin{abstract}
Background: The plant-pathogenic fungus Fusarium oxysporum f.sp.lycopersici (Fol) has accessory, lineage-specific (LS) chromosomes that can be transferred horizontally between strains. A single LS chromosome in the Fol4287 reference strain harbors all known Fol effector genes. Transfer of this pathogenicity chromosome confers virulence to a previously non-pathogenic recipient strain. We hypothesize that expression and evolution of effector genes is influenced by their genomic context.

Results: To gain a better understanding of the genomic context of the effector genes, we manually curated the annotated genes on the pathogenicity chromosome and identified and classified transposable elements. Both retro- and DNA transposons are present with no particular overrepresented class. Retrotransposons appear evenly distributed over the chromosome, while DNA transposons tend to concentrate in large chromosomal subregions. In general, genes on the pathogenicity chromosome are dispersed within the repeat landscape. Effector genes are present within subregions enriched for DNA transposons. A miniature Impala $(\mathrm{mimp})$ is always present in their promoters. Although promoter deletion studies of two effector gene loci did not reveal a direct function of the mimp for gene expression, we were able to use proximity to a mimp as a criterion to identify new effector gene candidates. Through xylem sap proteomics we confirmed that several of these candidates encode proteins secreted during plant infection.

Conclusions: Effector genes in Fol reside in characteristic subregions on a pathogenicity chromosome. Their genomic context allowed us to develop a method for the successful identification of novel effector genes. Since our approach is not based on effector gene similarity, but on unique genomic features, it can easily be extended to identify effector genes in Fo strains with different host specificities.
\end{abstract}

\section{Background}

The tomato pathogenic fungus Fusarium oxysporum forma specialis lycopersisci $(F o l)$ posses a two-partite genome. Eleven of the 15 chromosomes of the sequenced strain (Fol4287) are syntenic with chromosomes of the sister species Fusarium verticilloides and the more distantly related Fusarium graminearum, displaying high sequence similarity and conservation of gene order [1]. These core chromosomes contain all housekeeping genes and few transposable elements (TEs). Additionally,

\footnotetext{
*Correspondence: m.rep@uva.nl

'Plant Pathology, Swammerdam Institute for Life Sciences, University of Amsterdam, Science Park 904, 1098 XH, Amsterdam, the Netherlands Full list of author information is available at the end of the article
}

Fol4287 possesses four chromosomes that are devoid of housekeeping genes and accommodate $74 \%$ of the whole genome TE content and $95 \%$ of the class II TEs (DNA transposons). The four chromosomes and two smaller regions at the ends of two core chromosomes comprise the lineage-specific (LS) part of the $\mathrm{Fol}$ genome. The genes encoded in LS regions differ in their phylogenetic history from the genes on the core chromosomes [1,2]. The term lineage-specific (LS) reflects the largely clonal structure of the Fo species complex. Fo reproduces asexually and consists of many clonal lineages, which, if pathogenic, are grouped into host-specific formae speciales (ff. spp.) [3]. While some ff. spp. are monophyletic, others are composed of several clonal lineages that

\section{Biomed Central}


appear to have independently acquired the ability to infect the same host plant [4-6]. This polyphyletic origin was likely caused by horizontal transfer of chromosomes encoding host specific virulence genes between Fo lineages, thereby allowing the distinction of members of a f. sp., not by overall genetic relatedness, but by the presence or absence of certain LS chromosomes [1].

In Fol, one LS chromosome (chromosome 14 of Fol4287) largely defines the pathogenic phenotype of this f. sp., i.e. the ability to cause wilt disease in tomato. Horizontal transfer of this pathogenicity chromosome from a tomato pathogenic isolate to a non-pathogenic isolate during co-cultivation resulted in novel tomatopathogenic lineages, demonstrating that it contains genes that promote infection of tomato [1]. Among these genes are all known Fol4287 effector genes called SIX (Secreted In Xylem) genes. Like many other plant pathogens, Fol utilizes small, secreted proteins to promote virulence by manipulating its plant host and suppressing host defense responses, typically through interaction with host proteins $[7,8]$. Six proteins are small, commonly cysteine-rich, lack homology to other proteins and have a signal peptide for secretion [8]. Six of the seven previously described Six proteins are encoded on the pathogenicity chromosome; the genomic location of SIX4, whose gene product is recognized by the tomato resistance proteins I and I-1, is unknown because it is not present in the sequenced race 2 isolate Fol4287. Although SIX genes were likely acquired by horizontal transfer of the pathogenicity chromosome, they are not functionally independent of the core genome. Their expression requires the transcription factor Sge1 (SIX gene expression 1), which is encoded on a core chromosome [9]. It is unknown whether Sge1 regulates SIX gene expression directly or indirectly, for example through the action of other transcription factors.

Effector genes in other plant pathogens, such as Magnoporthe oryzae, Leptosphaeria maculans or Phythophthora infestans, are also found proximal to TEs and TEs have been proposed as the underlying agents that provide a plastic environment for the emergence of new virulence traits [10-12]. The potential of TEs to affect genome structure is a consequence of both their mobility and their inherent structure. Generally, two different $\mathrm{TE}$ classes are distinguished by their transposition intermediate: RNA or DNA. Class I TEs (or retrotransposons) transpose via a "copy-paste mechanism" by copying themselves into an RNA-intermediate before inserting at a new site, while class II TEs (or DNA transposons) leave the donor site to reintegrate at another site via a "cut-paste mechanism", although the original copy can also be retained [13]. Class I TEs are either flanked by terminal inverted repeats (TIRs), long terminal repeats (LTRs) or simple non-coding regions. Class II TEs are usually flanked by TIRs [14]. Special TE families are the MITEs (Miniature Inverted-repeat Tranposable Elements), non-autonomous class II TEs of short length, which are thought to have evolved from autonomous TEs by deletion of their transposase ORF [15]. Recombination between identical or highly similar TEs can cause structural rearrangements like deletions, inversions, duplications and translocations depending on the orientation and genomic location of the recombining TE members [16]. For an asexual fungus like Fol, TEmediated recombination might represent a mechanism to create genetic variation in the absence of meiotic recombination. Next to gross structural rearrangements, TEs also contribute to evolution of novel phenotypes by transposition into new sites. For example, insertion of the hAT transposase Drifter into the coding sequence of an ancestral SIX1 homolog $(S I X 1-H)$ disrupted the openreading frame (ORFs) of $S I X 1-H$, thus creating an effector pseudogene [8]. In another case, insertion of a Hornet-like transposon at the SIX4 locus of a japanese race $3 \mathrm{Fol}$ isolate created a fusion protein, which was no longer recognized by the corresponding I-1 tomato resistance protein [17]. TE insertion might also influence gene expression when it occurs within a promoter.

To further our understanding of the molecular basis of pathogenicity of Fol towards tomato, we conducted a detailed annotation of the predicted proteins encoded by the Fol pathogenicity chromosome. In addition, to advance our understanding of the potential role of the genomic context of effector genes in gene evolution or expression, we also annotated TEs on this chromosome. We thus obtained a detailed picture of the genomic landscape of the pathogenicity chromosome. Within this TE-rich landscape, we recognized mini-clusters of SIX genes. SIX genes are associated with two MITEs: a mimp upstream in all cases and, frequently, an mFot5 downstream. Using promoter deletions at two SIX gene loci, we studied the influence of the mimp on SIX gene expression. Finally, we were able to exploit the consistent presence of a mimp in the promoters of SIX genes and other virulence-associated genes to develop a method to identify candidate effector genes in F. oxysporum.

\section{Results}

Non-TE genes on the Fol pathogenicity chromosome group into a small set of functional classes

Non-TE ORFs occupy only $13 \%$ of the DNA space on the pathogenicity chromosome of $\mathrm{Fol}$, which consists of four supercontigs (sc) (sc 22, 36, 43, 51) in the most recent Fol genome assembly (Li-Jun Ma, personal communication, Table 1). Most of the manually curated 245 non-TE ORFs on this chromosome encode proteins of unknown function, which are annotated as hypothetical 
Table 1 Space occupied by TEs and non-TE ORFs on the pathogenicity chromosome

\begin{tabular}{lll}
\hline & In bp & Percent of sequence \\
\hline non-TE ORFs & 324923 & 13 \\
TEs & 581563 & 24 \\
total & 2457923 & 100 \\
\hline
\end{tabular}

proteins or proteins with domains of unknown function (140 ORFs, Figure 1). Some of these unknown proteins have homologous sequences in $F$. oxysporum or in other fungi (Additional file 1). Two functional groups stand out among the predicted products of the remaining 103 non-TE ORFs: secreted proteins (29) and proteins involved in secondary metabolism (35). Other functional groups include transcription factors (11), proteins with nucleic acid related functions (10), heterokaryon incompatibility (Het) proteins (4), transporters (3), cyclins (3) and other intracellular functions (17), such as GTPases and protein kinases (Figure 1). As reported by Ma et al., there are no genes for housekeeping proteins on the pathogenicity chromosome [1]. Among the predicted secreted proteins, we find nine secreted enzymes, such as oxidoreductase, chitinase and glucanase, and 20 secreted proteins of unknown function. Sixteen of the latter encode proteins smaller than 300 amino acids. Among those are the previously described effector genes SIX1, SIX2, SIX3, SIX5, SIX6 and SIX7 [18-20]. Proteins encoded on the Fol pathogenicity chromosome that are likely involved in secondary metabolism [1] include methyl transferases (6), cytochrome P450s (6) and glycosyltransferases (3). A putative secondary metabolite gene cluster on sc51 includes genes for three cytochrome P450s, a glycosyltransferase, a methyltransferase, a squalene-hopene cyclase and a homolog of Tri7, an acetyltransferase that is part of the trichothecene gene cluster in Gibberella zeae [21]. The genes in this putative secondary metabolite cluster are expressed during tomato infection (Additional file 2) and might therefore be important for pathogenicity of the fungus.

Currently, it is not known how F. oxysporum can transfer chromosomes horizontally from one strain to another. One hypothesis is that horizontal chromosome transfer (HCT) occurs via anastomosis tubes specialized, unbranched tubes that connect conidia or hyphae $[2,22]$. Anastomosis tubes result in heterokaryon formation between two fungal individuals [23]. This heterokaryon is only viable if the individuals have the same HET (Heterokaryon incompatibility) genotype; otherwise it undergoes a characteristic cell death reaction called an incompatibility reaction [24]. Four genes on the pathogenicity chromosome encode proteins with similarity to Het proteins in other fungi (FOXG_14188, FOXG_14292, FOXG_14283, FOXG_14284). Het proteins like Het-E from Podospora anserina often harbor NACHT (NAIP, CIIA, HET-E, TP1) domains, or

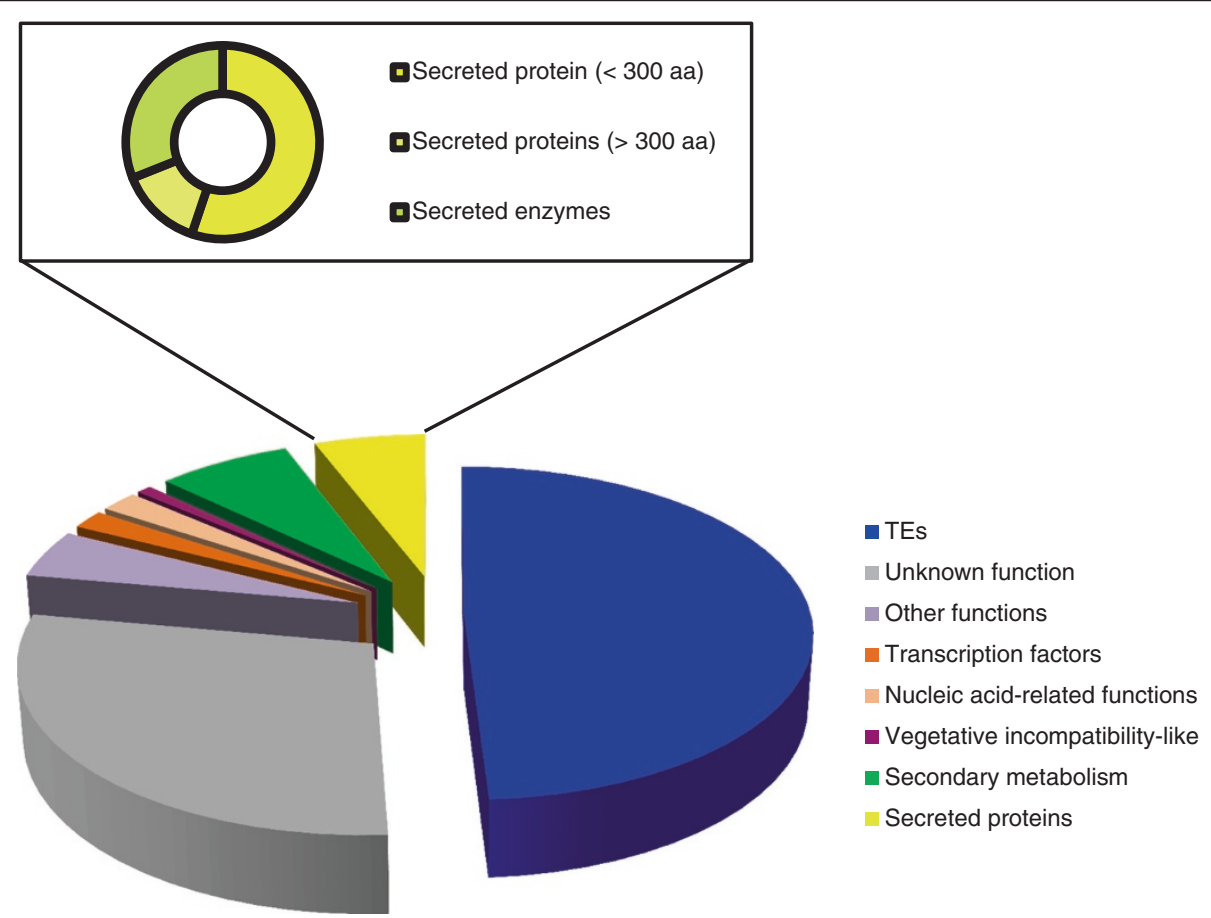

Figure 1 TEs dominate on the pathogenicity chromosome. TES and non-TE genes are presented as percentage of the total TE/gene content Genes coding for secreted proteins (including SIX genes) constitute one of the best-represented classes. 
a highly divergent nucleoside phosphorylase (Pfs) linked with protein-binding modules such as Ankyrin repeats [25]. FOXG_14188 encodes a protein with a NACHT domain, FOXG_14292 a protein with a Pfs domain and Ankyrin repeats, FOXG_14283 a protein with a Pfs, an ATPase and Ankyrin repeats and FOXG_14284 a protein with Pfs and Ankyrin repeats (Additional file 1). The presence of HET-like genes on the pathogenicity chromosome may be seen to contradict HCT via anastomosis tubes, because additional HET loci would raise the chance of incompatibility between strains, involving programmed cell death of fused compartments. On the other hand, incompatibility does not appear to be a barrier to HCT [1]. Moreover, we do not know whether the $H E T$-like genes on the pathogenicity chromosome are really involved in incompatibility.

Since transfer of the pathogenicity chromosome is sufficient to confer pathogenicity towards tomato, the virulence genes on it must be expressed in the new host strain. We know that there is crosstalk between the core and pathogenicity chromosome, because the core chromosome-encoded Sge1 controls SIX gene expression [26]. The presence of eleven genes encoding transcription factors on the pathogenicity chromosome suggests that transcription of genes on the pathogenicity chromosome may also be controlled by the chromosome itself. Among the transcription factors encoded on the pathogenicity chromosome are three copies of FTF1, which is induced upon plant infection [27], suggesting that at least a subset of the transcription factors encoded on the pathogenicity chromosome may be required for transcriptional reprogramming during plant infection.

Next to transcription factors, nine other genes encode proteins with nucleic acid-related functions (Figure 1). Most of these proteins are predicted to function in structural rearrangements of DNA or in chromatin modifications. FOXG_16427 encodes a poly(ADP)-ribose polymerase (Parp1) which binds to damaged or singlestranded DNA to recruit DNA repairing enzymes [28]. Other genes encode putative components of the RNA silencing machinery, including closely spaced genes for an RNA-dependent RNA polymerase (FOXG_16453), an RNA interference and gene silencing protein (FOXG_16455) and a RNaseH domain-containing protein (FOXG_16456). FOXG_14161 encodes a protein homologous to the eukaryotic conserved kinetochore protein Mis12 that is involved correct segregation of daughter chromatids during mitosis and meiosis [29]. FOXG_14165 encodes a protein with a BAH (bromoadjacent homology) domain which may interact with gene silencing components [30]. Similarly, FOXG_14186 encodes a chromodomain protein that typically recruits protein complexes to chromatin and reads the epigenetic code by recognizing lysine methylation [31]. Proteins involved in chromatin modification and RNA interference might influence gene expression during pathogenicity.

\section{The Fol pathogenicity chromosome harbours a large diversity of transposable elements}

To exhaustively identify TEs and TE relics on the Fol pathogenicity chromosome, we performed a selfBLASTN of the genome sequence, then identified multicopy sequences and sorted them into non-redundant families. Secondly, we looked for inverted repeats (IRs) of at least $19 \mathrm{bp}$ encompassing at most $5 \mathrm{~kb}$ of sequence. This expanded the set of identified TEs relative to an initial survey [1]. Taken together, TEs occupy about twice as much (24\%) chromosomal DNA space as nonTE ORFs (13\%, Table 1).

Both Class I and Class II TEs (full length and fragments) are present in approximately equal numbers (266 Class I, 249 class II, Table 2) on the pathogenicity chromosome, which is surprising because retrotransposons often dominate the TE fraction of a given genome [11,32-34]. For annotation of the TE classes we followed the classification system proposed by Wicker and colleagues that comprises both mechanistic and enzymatic criteria [14]. Class I TEs all transpose by transcribing themselves into an RNA intermediate, then reverse-transcribing the RNA by a TEencoded reverse transcriptase and inserting into a new genomic region. There are three orders of class I TEs: Long-terminal-repeat (LTR) TEs, long-interspersed nuclear elements (LINE) and short interspersed nuclear elements (SINE).

LTR retrotransposons are similar to retroviruses and encode multiple enzymatic domains including Gag (a viral coat protein), protease, $\mathrm{RNaseH}$, reverse transcriptase and integrase, flanked by long terminal repeats [14]. Within the LTR order we identified members of the Gypsy/Ty3 (27) and Copia/Ty1 (59) superfamilies, a novel class I TE named Yaret2, which encodes integrase (IPR001584), RNaseH (IPR012337), reverse transcriptase (IPR013103) and a Zinc-finger (IPR001878) domain, as well as two novel solo-LTR families. Solo-LTRs can be the result of intrachromosomal or intraelement recombination between the LTRs, thereby removing the internal domains and creating a solo LTR at the excision site [35]. Several of these LTR transposons have been previously recognized in Fo or in other pathogenic fungi. Nht2, for example, is also present on a LS chromosome of Fusarium solani [36].

LINE elements lack the LTRs that are characteristic for the retroviral-like class I TEs. In this order we identified 31 MGR583-like elements and 34 Yaret1 and Yaret1-like elements (24 and 10, respectively). MGR583 accompanies the effector gene AVR-PITA in some $M$. oryzae isolates [12]. The latter two are novel LINEs. Foxy (32 copies) represents the only TE of the SINE 
Table 2 Transposable elements on the Fol4287 pathogenicity chromosome

\begin{tabular}{|c|c|c|c|c|c|c|}
\hline \multicolumn{4}{|c|}{ Classification } & \multirow[t]{2}{*}{ Designation $^{1}$} & \multirow[t]{2}{*}{ Number } & \multirow{2}{*}{$\begin{array}{l}\text { Full length } \\
\text { number }\end{array}$} \\
\hline Order & Superfamily & Family & Number & & & \\
\hline \multicolumn{3}{|c|}{ Class I (retrotransposon) } & 237 & & 237 & 58 \\
\hline \multirow[t]{7}{*}{ LTR } & Gypsy/Tyз & & 24 & MAGGY-like retrotransposon (3 types) & 16 & 4 \\
\hline & & & & Skippy & 8 & 1 \\
\hline & Copia/Ty1 & & 55 & NHT2-like retrotransposon (5 types) & 51 & 1 \\
\hline & & & & Pcretro3-like retrotransposon & 4 & 1 \\
\hline & unclassified & & 20 & Yaret2 & 20 & 6 \\
\hline & solo-LTR & & 28 & Yaret2 solo-LTR & 12 & 12 \\
\hline & & & & Gollum (NHT2-like retrotransposon type 3 LTR) & 16 & 10 \\
\hline \multirow[t]{3}{*}{ LINE } & & & 68 & MGR583-like LINE element & 31 & 3 \\
\hline & & & & Yaret1 & 25 & 3 \\
\hline & & & & Yaret1-like & 12 & 0 \\
\hline SINE & & & 32 & Foxy & 32 & 10 \\
\hline unrelated & & & 10 & Marsu & 10 & 7 \\
\hline \multicolumn{3}{|c|}{ Class II (DNA transposons) - Subclass 1} & 208 & & 208 & 108 \\
\hline \multicolumn{2}{|l|}{ Crypton } & & 1 & FoCrypton & 1 & 1 \\
\hline \multirow[t]{17}{*}{ TIR } & Tc1/mariner & Pogo & 41 & Fot2 & 2 & 2 \\
\hline & & & & Fot3 & 7 & 2 \\
\hline & & & & Fot4 & 1 & 0 \\
\hline & & & & Fot5 & 23 & 6 \\
\hline & & & & Fot6 & 6 & 3 \\
\hline & & & & Fot8 & 2 & 0 \\
\hline & & $\mathrm{Tc1}$ & 3 & Impala & 3 & 0 \\
\hline & & hAT & 70 & Folyt1 & 3 & 3 \\
\hline & & & & Folyt2 & 1 & 0 \\
\hline & & & & Frodo & 5 & 3 \\
\hline & & & & Hornet & 16 & 7 \\
\hline & & & & Drifter & 1 & 1 \\
\hline & & & & NhORF4-like & 2 & 1 \\
\hline & & & & Sam & 1 & 1 \\
\hline & & & & YahAT1 & 6 & 5 \\
\hline & & & & YahAT2 & 9 & 4 \\
\hline & & & & YahAT3 & 3 & 1 \\
\hline
\end{tabular}


Table 2 Transposable elements on the Fol4287 pathogenicity chromosome (Continued)

Нор3

Hop4

Hop5

Hop6

mimp (unclassified)

mimp1

$\operatorname{mimp2}$

$\operatorname{mimp} 3$

$\operatorname{mimp} 4$

mFot5

Gimli

\section{Class II (DNA transposons) - Subclass 2}

9

Class II (DNA transposons) - unclassified

Helitron

40

total number of TEs

494

unclassified

2
4
7
10
1
6
5
2
6
2
24
7
6
17
14

1

1

5

2

2

24

7

6

designations in bold letters indicate TEs that have been described in Fusarium oxysporum before. 
class on the pathogenicity chromosome. Foxy appears to be an active TE that is specific for Fusarium species [37]. Foxy elements are the most abundant class I TEs in Fol and they are evenly distributed over the pathogenicity chromosome and also throughout core chromosomes [1,38] (this manuscript). This dispersed distribution pattern is also apparent for the other class I TEs. Finally, we detected 10 copies of Marsu, which is a retrotransposon that cannot be classified as LTR, LINE or SINE. Copies of Marsu were first described in Fo f. sp. phaseoli where they were found downstream of the FTF1 gene [27]. Ramos et al. speculated that the Marsu element might be responsible for gene duplication events of FTF1 [27]. For most retrotransposon classes on the pathogenicity chromosome, we find only few fulllength copies. Marsu is the marked exception: seven of the ten copies are full-length. Marsu copies are present in other Fol4287 LS regions, and two copies reside on core chromosomes. Although we did not detect identical copies within the genome sequence of Fol4287, the presence of moderately divergent copies and many full-length copies suggest that Marsu elements have been active relatively recently.
Compared to class I elements, class II elements are less evenly distributed on the chromosome and many aggregate in large chromosomal subregions (Figure 2). Class II elements are divided into two subclasses. Among subclass I we identified one Crypton copy. Cryptons encode a tyrosine-recombinase to cut and rejoin recombining DNA strands. They were first identified in human pathogenic fungi and were later found to be domesticated in vertebrates $[39,40]$. There are more Crypton copies present on other LS chromosomes, but none on core chromosomes. Within subclass II we identified nine Helitron copies. Helitrons are unusual class II TE; instead of a 'cut and paste' mechanism they transpose via a rolling-circle mechanism [14]. With this transposition mechanism they often capture host genes and thus contribute to genome evolution [15]. At least eight of the nine Helitron copies on the pathogenicity chromosome are intact; one is truncated by a sequence gap (Additional file 1). All copies are $99-100 \%$ identical in sequence, and there are intact Helitron copies on core chromosomes, suggesting that Helitrons are still active.

The best-represented order of class II TEs are the Terminal Inverted Repeat (TIR) TEs (Table 2). These TEs

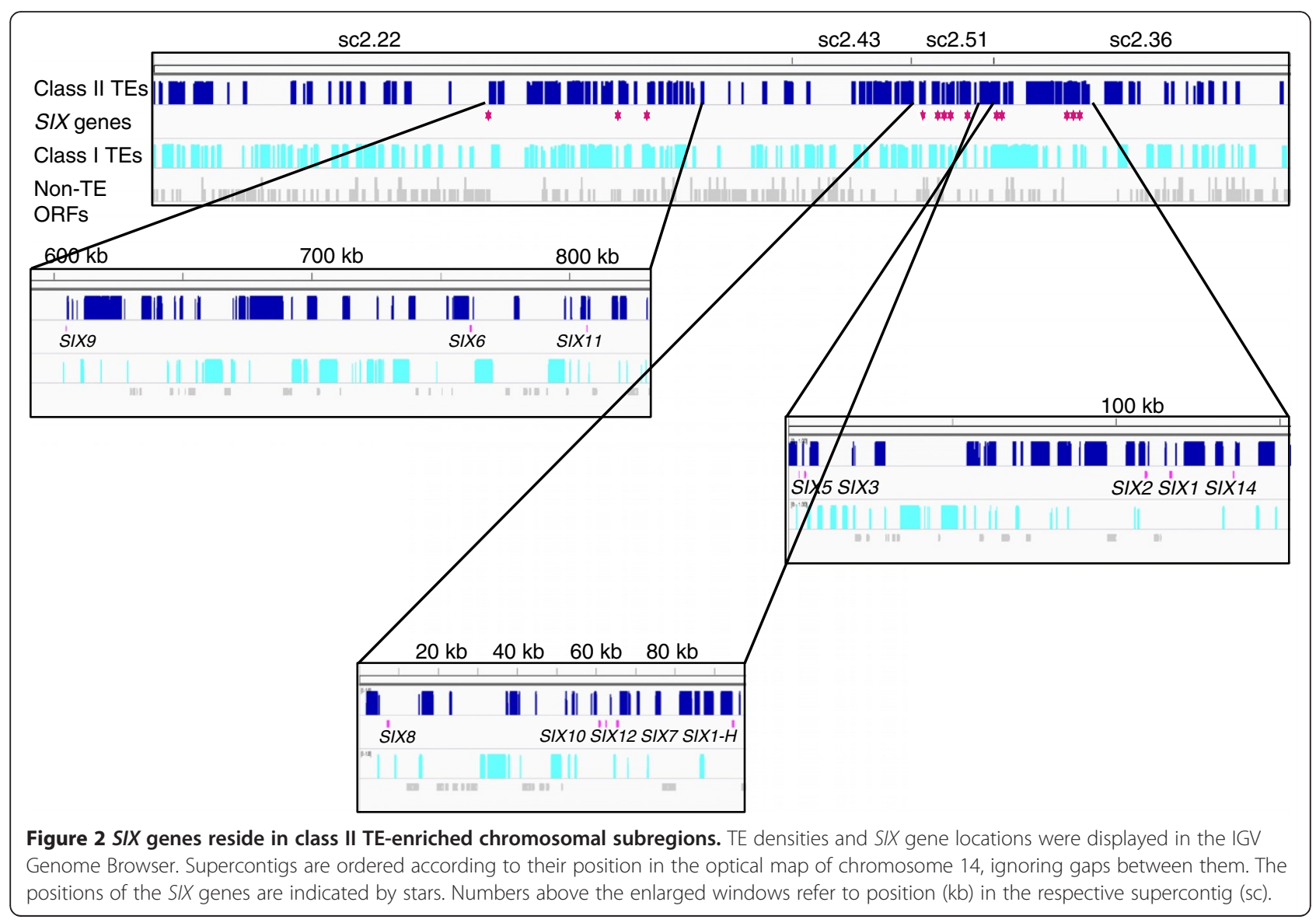


consists of a transposase ORF flanked by TIRs [14]. Among the TC1/mariner superfamily, we found multiple, diverse Fot lineages belonging to the pogo family. This finding confirms the previously shown preferred localization of pogo elements on LS chromosomes [41]. We observed a similar diversification of Hop elements belonging to the Mutator family. Five Hop classes are present with one to 13 copies, most of which are not full-length, although Hop has been shown to be active in Fo [42]. Most TE families, including three Folyt copies and 16 Hornet copies, belong to the hAT family. Folyt has been identified as an expressed and active transposable element in Fol by transposon trapping [43]. Hornet1 was discovered during analysis of transposons in Fo f. sp. melonis [44]. The only copy of the hAT transposon Drifter adjoins the truncated effector gene SIX1- $H$ [45]. Overall, as previously shown for some genomic regions in Fom, class II TEs seem to preferentially insert into or close to each other, creating class II TE-enriched subregions on the Fol pathogenicity chromosome.

These subregions are also enriched for MITEs. MITEs are non-autonomous TEs, which basically consist of TIRs flanking a short non-coding DNA sequence. Three different classes of MITEs are present on the pathogenicity chromosome: 55 mimps (miniature Impalas), three Gimlis and 14 mFot5s (of which one is interrupted by a retrotransposon). MITEs require an associated transposase for transposition. Often, this associated transposase has similar TIRs [15]. For mFot5 transposition, two TEs encoding intact Fot 5 transposases on the pathogenicity chromosome might facilitate transposition. Mimps are transposed by the Impala transposase, which was shown to be active in the melon pathogenic strain Fo f. sp. melonis by transposon tagging [46,47]. However, in Fol4287 all three Impala copies, which reside on the pathogenicity chromosome, do not encode a full-length transposase, suggesting that mimps are presently not actively transposed in Fol4287. The large diversification of the mimp lineages with members of more than four families and without two identical copies also suggests that mimps are not presently active in Fol4287.

\section{Mimps are associated with promoters of SIX genes}

SIX genes tend to reside in chromosomal subregions that are enriched for class II TEs, sometimes as miniclusters (Figure 2, Additional file 1). For example, SIX1 and SIX2 form a mini-cluster with one intervening gene (salicylate hydrolase homolog (SSH1)) and two intervening mimps, flanked by another mimp and a Fot5 (Figure 3, see below). SIX3 and SIX5 form another minicluster with an intervening mimp, with nearby mFot5 and Fot 5 fragments. This mini-cluster is flanked on both sides by inverted repeats, suggesting that this mini- cluster might be able to be transposed (Figure 3, Additional file 1).

A closer inspection of the SIX gene promoters, which we pragmatically define as 1500 bp upstream of the start codon, revealed the presence of a mimp in the promoters of SIX1,SIX2, SIX3, SIX5, SIX6 and SIX7 (Figure 4). The mimp in the SIX1 locus was revealed by re-sequencing, because in the Fol4287 genome assembly there is a sequence gap upstream of the SIX1 ORF. Another sequence gap separates a mimp from SIX7. We were not able to bridge this gap by PCR and therefore cannot rule out that the distance between the mimp and SIX7 is bigger than $1.5 \mathrm{~kb}$ or that there is another mimp present that is closer to the SIX7 start codon. The avirulence gene $S I X 4 / A V R 1$ of race 1 Fol strains, which is not present in Fol 4287 (race 2), also harbors a mimp in its promoter sequence (Figure 4). The pathogenicity chromosome harbors more than half of the mimps present in the Fol4287 genome (Table 3). The other copies are mainly present on the three other LS chromosomes with the exception of four mimps on core chromosomes, as observed before [48]. Only a subset of the mimps on the pathogenicity chromosome is present in putative promoters (i.e. within $1500 \mathrm{bp}$ of a predicted start codon). While SIX1-7 all harbor a mimp in their promoter, only $8.3 \%$ of all annotated non-TE ORFs on the pathogenicity chromosome do so. This association of mimps with SIX gene promoters is highly significant (chi-square test $\mathrm{p}=5.25 \mathrm{E}-16$ for association by chance of mimps with the six known SIX genes on the Fol4287 pathogenicity chromosome). Additional annotated ORFs with a mimp in the promoter region encode a bZIP transcription factor, an integral membrane protein, an alpha$\mathrm{N}$ glucosaminetransferase, the Ftf1 transcription factor (2 copies), a catalase-peroxidase, the oxidoreductase Orx1, a homolog of the Verticillium dahliae avirulence protein Ave1, a methyltransferase, a cytochrome P450 and a squalene-hopene cyclase. The latter three genes belong to the putative secondary metabolite cluster that is co-expressed during plant infection (see above). Likewise, FTF1 has previously been shown to be expressed during plant infection [27]. The catalase-peroxidase and Orx1 are secreted in the xylem sap of Fol-infected tomato plants [19] (this manuscript). Overall, therefore, mimps seem to be preferentially associated with the promoters of genes that are expressed during plant infection.

To see whether additional, potentially regulatory elements may be enriched in $S I X$ gene promoters, we analyzed the promoter sequences of SIX1,SIX2, SIX3, SIX5, SIX6 and SIX7 for enriched k-mers. Several overlapping 6 to 9 mers were significantly enriched within these promoters. The most frequent of these form the sequence TCGGCAGTT (see Methods for details). Perfect matches to this sequence 


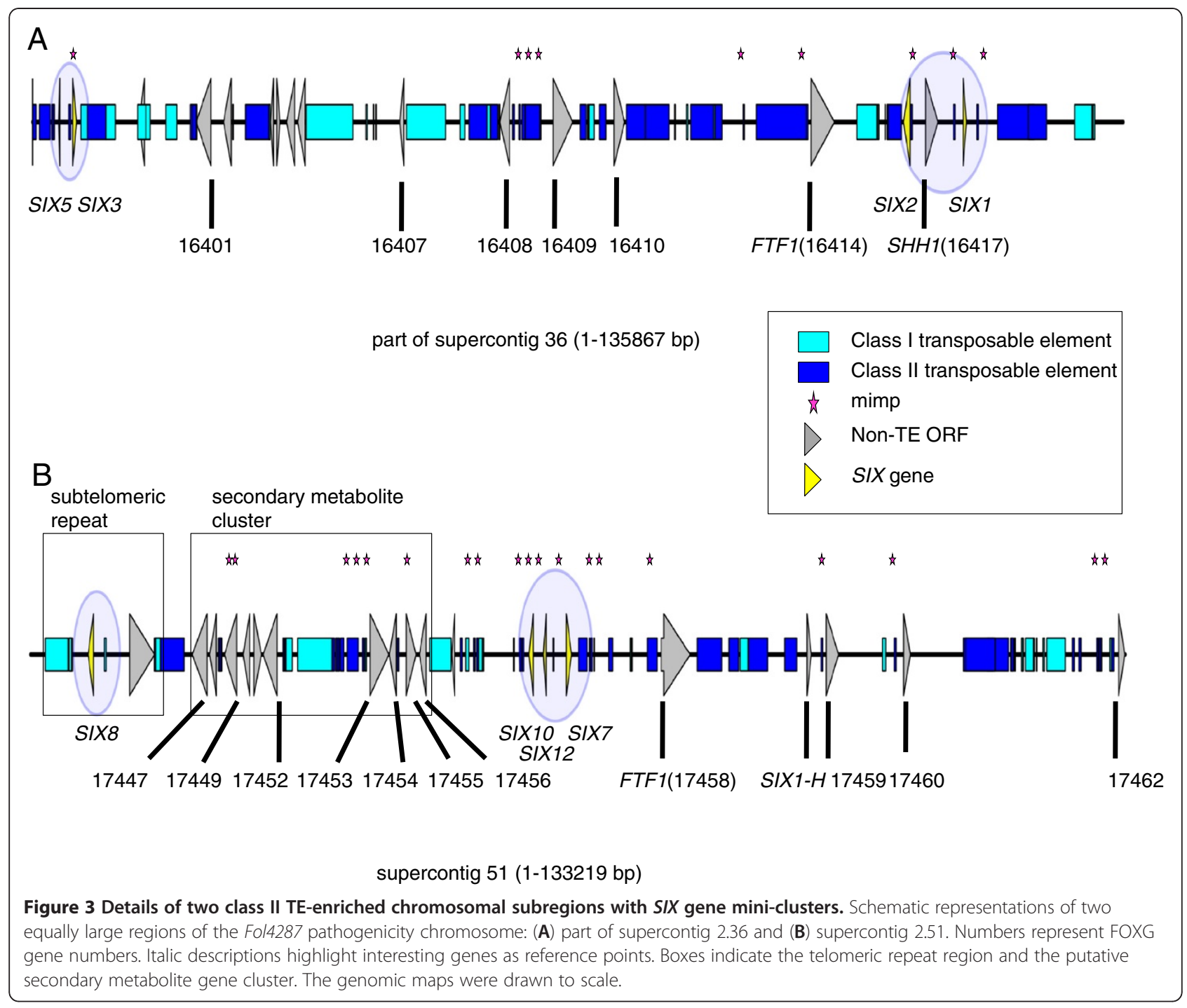

are present in the SIX1 and SIX3/SIX5 promoters. Compared to the entire gene set of the Fol4287 genome, the association between the presence of at least one or two of the 6mers TCGGCA, GGCAGT and the 7mer GGCAGTT and the $1000 \mathrm{bp}$ upstream region of effector genes appears to be significant (Additional file 3).

Finally, we also examined the 1500 bp downstream of the STOP codons of SIX genes. mFot5 is present downstream of SIX2, SIX4, SIX5 and SIX7 (Figure 4). The association of this MITE with the SIX genes is weaker than the mimp association with the SIX gene promoters, because it is not present downstream of all the SIX genes on the pathogenicity chromosome.

\section{SIX1 gene expression is not dependent on the presence of a mimp in the promoter}

We next wanted to know whether the mimp or the putative regulatory elements enriched in the SIX gene promoters are directly involved in transcriptional regulation of the SIX genes. To test this, we designed two constructs to replace different parts of the SIX1 promoter with a hygromycin resistance cassette. Both deletion constructs included the mimp, the difference between the constructs being that the SIX1p1189 construct (1552 to 363bp upstream of the translation start site) deletes only three of the six conserved SIX gene promoter 12 mers, while the SIX1p1230 construct $(-1552 \mathrm{bp}$ to $-323 \mathrm{bp})$ deletes five of these 12 mers (Figure 5A).

First, we tested whether SIX1 was still expressed in the promoter deletion strains in vitro. Most SIX genes are not highly expressed in vitro, their expression is only switched on upon plant infection. However, a low amount of SIX1 transcript is detectable in vitro [26]. To our surprise, SIX1 was expressed in both SIX1p1189 and SIX3p1230 promoter deletion strains despite the absence of a large part of the SIX1 upstream region (Figure 5B). 


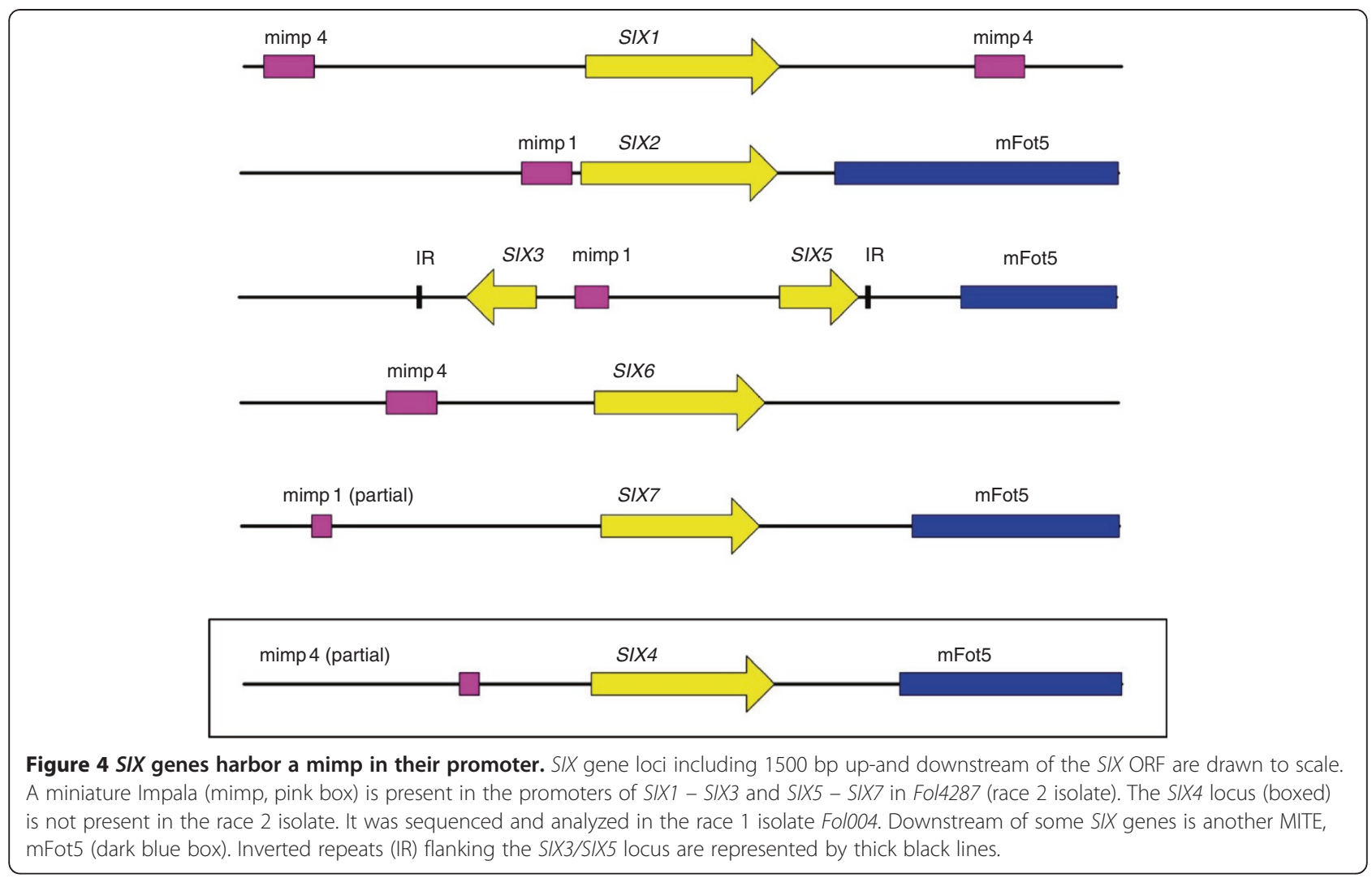

Six 1 is recognized by the tomato resistance protein I-3 and triggers disease resistance in tomato plants carrying the I-3-gene, thereby prohibiting extensive fungal infection [49]. Upon plant infection, SIX1 was only expressed from strains with the shorter SIX1p1189 deletion in both susceptible and resistant tomato cultivars. All transformed strains remained pathogenic towards tomato without Fol resistance genes, indicating that they were not affected in pathogenicity (Figure 5D). Consistent with the in planta expression pattern, only the wild type and strains with the SIX1p1189 promoter deletion were avirulent on the resistant tomato cultivar. In contrast, I-3 tomato cultivars that were infected with Fol strains carrying the SIX3p1230 promoter deletion were diseased, indicating the absence or reduced accumulation of the Six1 avirulence protein (Figure 5D). Taken together, deletion of the mimp did not impair SIX1 expression in vitro or in in planta and this mimp is therefore not required for transcriptional regulation of the SIX1 gene. However, a promoter region including two TCGGCA elements appears to be required for SIX1 expression during plant infection.

\section{SIX3/SIX5 promoter deletions reveal complex regulation at this locus}

To further investigate the functional role of mimps in effector gene expression, we also designed promoter deletion constructs for the SIX3-SIX5 locus. SIX3 and SIX5 share the same 1365 bp upstream sequence. This bidirectional promoter allowed us to test the expression of two different SIX genes with the same promoter deletion constructs. Like SIX1, SIX3 is also recognized by a tomato resistance protein, I-2 in this case, and expression of SIX3 and SIX5 is low but detectable in vitro $[18,26]$. We designed three promoter deletion constructs: SIX3p539 (1095 to 520 bp upstream of the transcription start site), SIX3p807 (-1095 to $-252 \mathrm{bp}$ ) and SIX3p859 (-1059 to $-200 \mathrm{bp).} \mathrm{SIX3p539} \mathrm{deletes} \mathrm{six}$ of the nine TCGGCA elements, but does not include the mimp, SIX3p807 includes the six TCGGCA elements and the mimp and SIX3p859 additionally deletes one more TCGGCA element (Figure 6A). Again, none of these promoter deletions impairs expression of SIX3 or SIX5 in vitro (Figure 6B). During plant infection, a reduced level of SIX3 mRNA was detected in Fol strains carrying the SIX3p539 deletion, but not in strains with

Table 3 Distribution of mimps in the Fol4287 genome

\begin{tabular}{lll}
\hline & Number of mimps & Mimp per $\mathbf{M b}$ \\
\hline pathogenicity chromosome & 54 & 21,14 \\
other LS chromosomes & 45 & 3,26 \\
core chromosomes & 4 & 0,09 \\
\hline
\end{tabular}




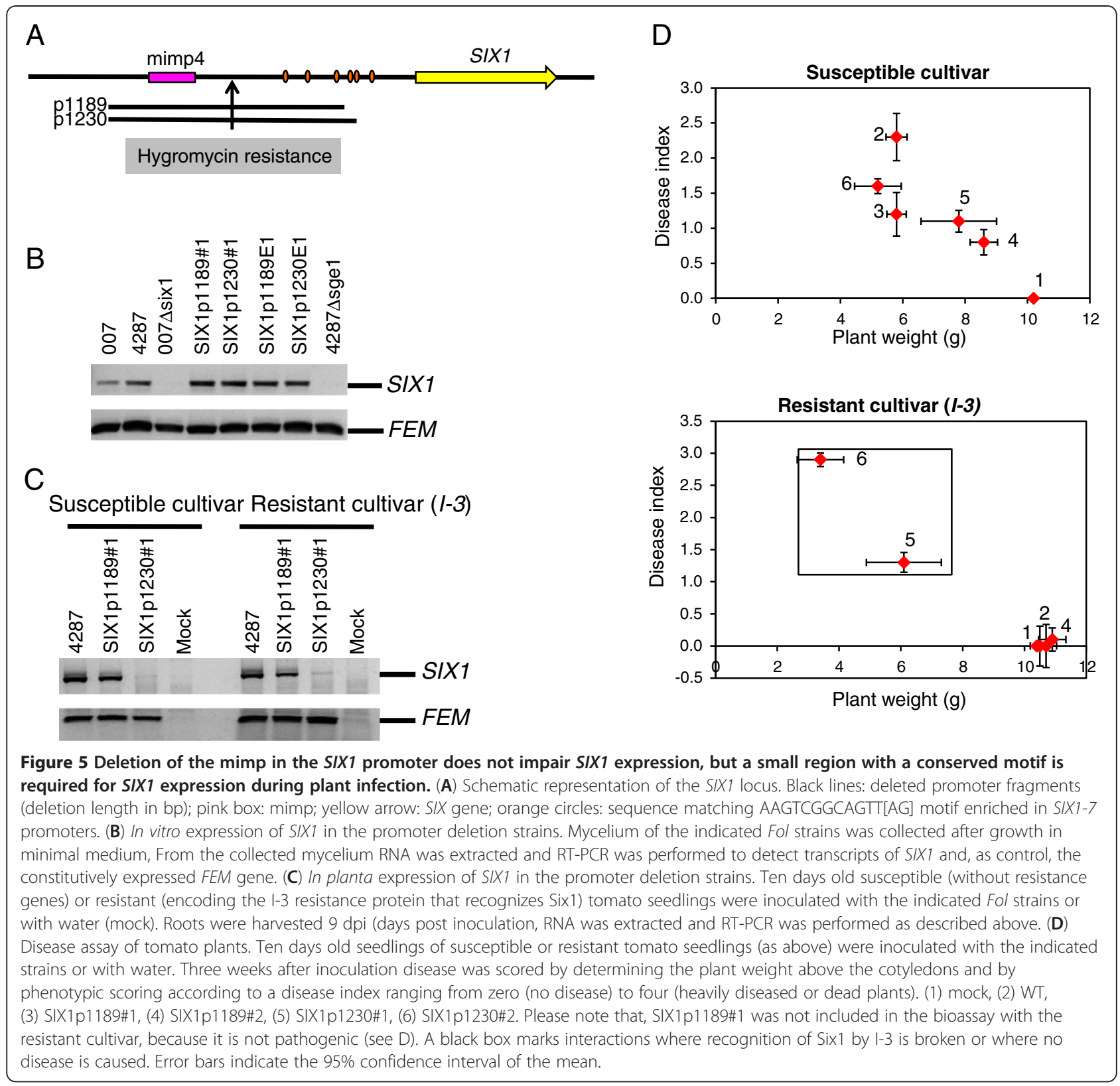

the SIX3p807 deletion. SIX5 is not expressed in either SIX3p539 or SIX3p807 deletion strains (Figure 6C). Remarkably, both SIX3 and SIX5 are expressed during plant infection in $\mathrm{Fol}$ strains carrying the most extensive promoter deletion, SIX3p859 (Figure 6C). With one exception, all tested strains were still able to cause disease on susceptible tomato cultivars and are thus not generally impaired in pathogenicity (Figure 6D). Only strains with the SIX3p859 deletion trigger a resistance response in tomato plants carrying the $I-2$ resistance gene, while the Fol strains with the SIX3p539and SIX3p807 promoter deletions break I-2-mediated resistance, con- sistent with the Six 3 protein not being produced by these strains (Figure 6C). Although in the Fol strains carrying the SIX3p539 promoter deletion a residual amount of SIX3 transcript is present, these strains are virulent. This may be explained by the additional requirement of SIX5 for I-2-mediated resistance (manuscript in preparation).

From this set of experiments in two SIX gene loci, we can conclude that the mimps are not required for regulation of SIX gene expression. On the other hand, deletion of a short region containing a single TCGGCA element in the promoter of SIX1 abolishes SIX1 


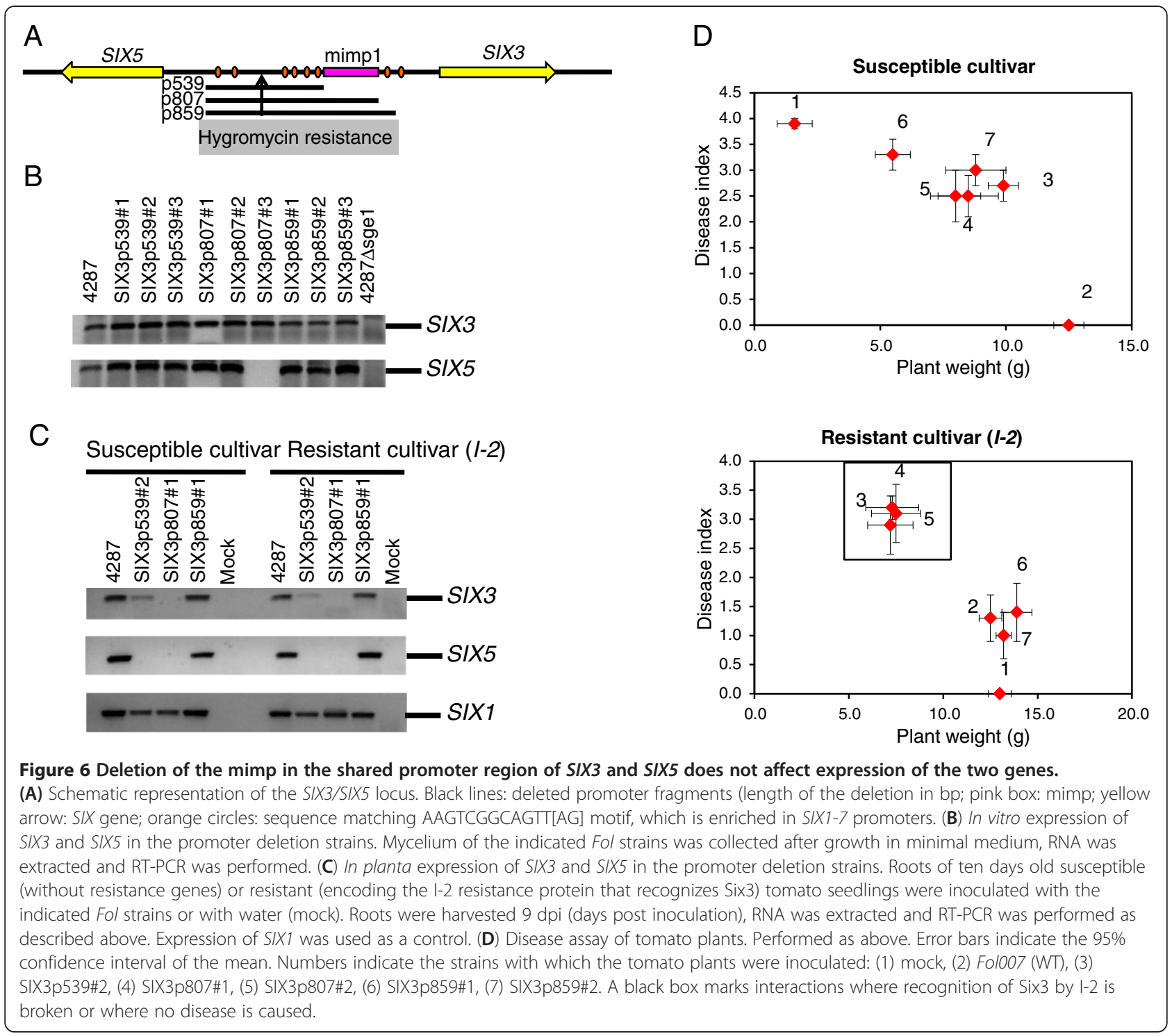

expression suggesting that this motif might represent a transcription factor-binding site (Figure 5C). In contrast, at the SIX3-SIX5 locus additional deletion of a region containing the same motif restores expression of both SIX3 and SIX5 during plant infection (Figure 6C).

The presence of mimps in the promoters of SIX genes enables prediction of novel effector candidates

Next, we wanted to test whether we can use the consistent presence of a mimp in the upstream region of the SIX genes to predict novel effector candidates. We searched the Fol4287 genome for the presence of a mimp TIR within $2 \mathrm{~kb}$ upstream of an ORF encoding a protein with an $\mathrm{N}$-terminal signal peptide for secretion (as defined by SignalP). We also analyzed the xylem sap proteome of Fol-infected tomato plants by mass spectrometry to see which of the predicted effectors are secreted by the fungus during plant infection.

By the in silico search for mimp-association we predicted 16 effector genes in Fol4287, which are located on chromosomes 3, 6 and 14. These include three of the known SIX genes on the pathogenicity chromosome: SIX2, SIX3, SIX6. SIX1 and SIX7 were not identified because of sequencing gaps in the Fol4287 genome assembly (see above). SIX5 is a small gene comprising three exons. The first exon is unusually short and ends directly after the encoded signal peptide for secretion. Therefore, SIX5 escaped signal peptide prediction (by SignalP) and thus was not identified with our approach.

Besides the known SIX genes, we identified nine genes coding for small secreted proteins and four genes coding for secreted enzymes with a mimp in the upstream region (Table 4). The latter comprise several multi-copy 
Table 4 Novel effector candidates identified by searching for genes with a mimp IR in their promoter

\begin{tabular}{|c|c|c|c|c|c|c|}
\hline \multicolumn{3}{|l|}{ Gene description } & \multicolumn{2}{|c|}{ Mimp in promoter ${ }^{1}$} & \multirow[t]{2}{*}{$\mathrm{SP}^{2}$} & \multirow{2}{*}{$\begin{array}{l}\text { Protein } \\
\text { in } \\
\text { xylem } \\
\text { sap }\end{array}$} \\
\hline Encoded protein & Chromosome & FOXG or genomic location & $\begin{array}{l}\text { Identified in } \\
\text { search }\end{array}$ & $\begin{array}{l}\text { Distance mimp IR-ATG } \\
\text { [bp] }\end{array}$ & & \\
\hline \multicolumn{7}{|l|}{ SIX genes } \\
\hline Six1 (corrected) & 14 & FOXG_16418 (incorrect) & $n o^{3}$ & 1192 & yes & yes \\
\hline Six 2 & 14 & FOXG_16416 & yes & 211 & yes & yes \\
\hline Six3 & 14 & FOXG_16398 & yes & 232 & yes & yes \\
\hline Six 5 & 14 & SC36[3273-3407] & $n o^{4}$ & 1132 & yes & yes \\
\hline Six6 & 14 & FOXG_14246 & yes & 668 & yes & yes \\
\hline Six7 & 14 & SC51[65216-65875] & no & (sequence gap) & yes & yes \\
\hline \multicolumn{7}{|c|}{ Novel effector candidates } \\
\hline Six8 & 14,14 & FOXG_17445, FOXG_16464 & no ${ }^{4}$ & 109 & yes & yes \\
\hline Six8b & $3,3,6,6$ & $\begin{array}{l}\text { SC18[1122404-122824],SC18[862700-863120], SC41 [221648-222068], SC21[219855- } \\
\text { 220275] }\end{array}$ & yes & 1026, 1972 & yes & no \\
\hline Six9 & 14 & FOXG_14223 & yes & 249 & yes & yes \\
\hline Six10 & 14 & FOXG_17457 & $n o^{4}$ & 384 & yes & yes \\
\hline Six11 & 14 & SC22[806692-807024] & yes & 322,852 & yes & yes \\
\hline Six12 & 14 & SC51[62415-62753] & $n o^{5}$ & 837 & no & yes \\
\hline Six13 & 6,6 & FOXG_17131 (5' extended), SC42[126863-127192] & yes & 1971 & yes & yes \\
\hline Six14 & 14 & SC36[135867-136180] & yes & $211,258,681,1215$ & yes & yes \\
\hline FoAve1 & 14 & SC36[201730-202101] & yes & 788 & yes & no \\
\hline $\begin{array}{l}\text { conserved secreted } \\
\text { protein }\end{array}$ & 14 & FOXG_14254 & yes & 1312 & yes & no \\
\hline secreted protein & 15 & SC38[202206-202388] & yes & 1717 & yes & no \\
\hline secreted protein & 14 & SC51[127492-128836] & yes & 1236 & yes & no \\
\hline \multicolumn{7}{|l|}{ Secreted enzymes } \\
\hline Orx1 & 14 & FOXG_14258;FOXG_14236 & yes & 554 & yes & yes \\
\hline catalase-peroxidase & 6,14 & FOXG_17130, FOXG_17460 & yes & 921 & yes & yes \\
\hline metalloprotease & 3,6 & SC47[78991-79260], SC42[41025-41294] & yes & 394 & yes & no \\
\hline
\end{tabular}

distance between the mimp IR and the ATG start codon

predicted signal peptide.

not identified because of a sequence gap in the genome assembly.

not identified because of a short first exon.

not identified due to absence of a signal peptide. 
genes in the Fol4287 genome: two (non-identical) ORX1 copies, two copies of a gene coding for a catalaseperoxidase, two copies of a gene coding for a metalloprotease and three copies of a gene coding for an endo-polygalacturonase (Table 4). Both Orx1 and the catalase-peroxidase proteins were identified with mass spectrometry in the xylem sap of Fol-infected tomato plants, suggesting that they may play a role during plant infection.

Next to these two enzymes, we obtained protein sequences for four of the nine predicted effector proteins from the xylem sap proteome. Additionally, we identified three more small proteins in the xylem sap of infected tomato plants that were not predicted by our in silico search. We named the genes for which we found the protein products in xylem sap SIX8 - SIX14; one additional gene we named $S I X 8 b$ for its high similarity to SIX8 (Table 4). Upon inspection of the regions upstream of their respective genes we could always identify a mimp. SIX8, SIX10 and SIX12 were not found with the in silico search because no signal peptide was detected. Similar to SIX5, SIX8 and SIX10 have a short first exon and therefore the signal peptide was not recognized by SignalP. SIX12 is an unusual effector gene: it does not encode a protein with a canonical signal peptide for secretion.

In contrast to the other SIX genes in Fol, SIX8 is not a single gene, but is present in two copies on the pathogenicity chromosome (sc36 and sc51) and in subtelomeric regions on chromosomes 2, 3 and 7 in a repeated block of around $7400 \mathrm{bp}$. This block includes incomplete copies of the class II TEs Marsu and YahAT7, a Foxy and a gene encoding an unknown protein (Figure 3, Additional file 1). The repeated sequences flanking the SIX8 genomic block on sc36 suggest that SIX8 is present in a subtelomeric region. Furthermore, two copies of a related gene, SIX8b, are present on chromosomes 3 and 6 each in the Fol4287 genome. In total, there are nine SIX 8 and four SIX8b copies in the Fol4287 genome sequence. Both SIX8 and SIX8b appear to be preceded by a complex structure of (partial) mimps and mimp IRs (Additional file 4).

Like the SIX1-7 genes described above, the newly identified SIX genes, as well as several additional potential effector genes for which we did not find evidence for expression in planta, reside in class II TE-rich subregions (Figure 2, Table 4, Additional file 1). SIX11 resides in a region that includes $S I X 6$, three genes coding for conserved secreted proteins, one gene for a MFS transporter and one for a fumarate reductase/succinate dehydrogenase, a FTF1 homolog and the ORX1 gene. SIX14 is part of a cluster containing SIX1, SSH1 and SIX2 (Figure 3). SIX10 and SIX12 make up a mini-cluster with SIX7. Similar to the SIX3/SIX5 mini-cluster, SIX12 is flanked on both sides by inverted repeats, suggesting that it may be mobilized by a transposase that recognizes these IRs. SIX13 (FOXG_17131 - 5' extended) is part of a duplicated region on chromosome 6 (sc 42), which is different from the interchromosomal duplication shared with chromosome 3 .

Taken together, we have developed a method to predict novel effector genes in genomes of $F$. oxysporum based only on the following characteristics: (1) coding for small, secreted proteins, (2) harboring mimps or inverted repeats of mimps within $2 \mathrm{~kb}$ upstream of the start codon. We validated this method by mass spectrometric analysis of the xylem sap of Fol-infected tomato plants and confirmed in planta secretion of several predicted novel candidate effectors. These novel SIX genes represent ideal candidates for functional analysis.

\section{Discussion}

Effector genes on the Fol pathogenicity chromosome are associated with chromosomal subregions enriched in class II transposable elements

TEs dominate the Fol pathogenicity chromosome with large aggregates of class II TEs and more evenly distributed class I TEs. Interspersed within this TE-rich landscape are mostly single non-TE ORFs, a putative secondary metabolite cluster and the SIX gene miniclusters. In many plant and fungal species with expanded genomes, retrotransposons are mainly responsible for genome expansion. Their mode of replication, which involves creating new copies during every transposition cycle, can rapidly increase genome size. Often, a single or few class I TEs account for the majority of TEs present in a genome. The maize genome, for example, consists of $76 \%$ class I TEs, with the Gypsy family element huck and the Copia element ji together accounting for nearly one quarter of the genome sequence [33]. Similarly, in the obligate fungal pathogen Blumeria graminis $\mathrm{f}$. sp. hordei, the class I TE I (Line/Sine) alone occupies $17.2 \%$ of the entire genome space [32]. On the Fol pathogenicity chromosome, we do not observe such a massive expansion of retrotransposons. Instead, large aggregates of class II TEs are associated with genes involved in pathogenicity, such as the SIX gene miniclusters. The tendency of class II TEs to concentrate in subchromosomal regions might result from recombination of their IRs with IRs of the same or a similar TE family. Occasionally, SIX genes might be trapped between the IRs and subsequently transposed together with the TE, resulting in the observed presence of SIX genes within class II TE-enriched chromosomal subregions. Support for this hypothesis stems from the observation that IRs directly flank SIX12 and the SIX3/ SIX5 mini-cluster, although the transposase recognizing these IRs remains unknown. Similarly, the highly dynamic genomic location of the small, subtelomeric gene family AVR-Pita within the M. oryzae population has been attributed to the retrotransposons Inago-1 and 
Inago-2, which flank AVR-Pita. These are thought to be involved in multiple translocation events of AVR-Pita, thereby facilitating a cycle of loss and gain of recognition by rice cultivars encoding the cognate Pita resistance protein [12]. Next to retrotransposons, some DNA transposons have also been observed proximal to fungal effector genes. In Leptosphaeria maculans, putative effectors are clustered in AT-blocks together with three significantly over-represented TEs (one class I and two class II) [11]. Clustering of virulence genes might provide a selective advantage, because all captured genes experience the same genomic environment, e.g. an open or closed chromatin structure, thereby being simultaneously amenable for transcriptional regulation [50]. This might facilitate coordinated gene expression during plant infection.

\section{MITEs and Fol evolution}

Mimps are always found within $1500 \mathrm{bp}$ upstream the translation start site of SIX genes as well as upstream of several other genes that are expressed during plant infection. Mimps are uniformly small in size, ranging from 200 - 220 bp. Their central region has no coding capacity and is flanked by $\sim 27$ bp TIRs that resemble the TIRs of the Tc1/mariner transposase Impala [51]. Impalas have been shown to transpose mimps in a heterologous system [52]. However, none of the Impala copies in the Fol genome are intact, suggesting that mimps are not currently transposed in Fol4287. In the past there appear to have been several bursts of mimp amplification resulting in at least six mimp subfamilies present in Fol [51]. Strikingly, more than half of the mimps in Fol4287 are present on the pathogenicity chromosome and the other mimps, with four exceptions, are restricted to the LS regions (Table 3) [51].

mFot 5 s reside downstream of the $S I X 1 / S S H 1 / S I X 2$, the SIX3/SIX5 and the SIX10/SIX12/SIX7 mini-clusters as well as downstream of the solo SIX9 gene (Figure 3, Additional file 1). mFot5 is also part of the putative secondary metabolite cluster that is co-expressed during Fol infection of tomato plants (Figure 3, Additional file 2). Downstream of SIX11 is no mFot5, but a full-length Fot5. The same is true for ORX1, which encodes an oxidoreductase that is secreted by Fol during tomato infection. mFot5 is a pogo-like MITE, less than $500 \mathrm{bp}$ long with TIRs similar to those of the Fot 5 transposon. In contrast to the lack of intact Impalas for mimp transposition, Fol4287 possesses around 64 intact Fot5 transposase ORFs that could mobilize mFot5s [41].

\section{What could be the function of mimps in promoters of effector genes?}

Strikingly, mimps are not only present in SIX gene promoters, but also in the promoters of several other genes that are expressed during plant infection. Among these are the gene for the oxidoreductase Orx1 and two genes of the presumptive secondary metabolite gene cluster. One possible scenario is that the mimp is a domesticated TE, which has adopted a function as transcription factor binding site, perhaps for Sge1, the transcription factor regulating SIX gene expression [26]. We tested this by deleting fragments of varying length in the promoter of SIX1 and the bidirectional, shared promoter of SIX3 and SIX5. In a strain in which the mimp in the promoter of SIX1 was deleted (Fol4287SIX1p1189), SIX1 expression in vitro and in planta was the same as in wild type. Likewise, SIX3 and SIX5 expression was not affected in a strain in which the mimp was absent in their shared upstream region (Fol4287SIX3p859). Therefore, we can rule out a direct involvement of the mimp in transcriptional regulation of SIX gene expression.

We did, however, observe that the presence or absence of other promoter regions affect gene expression at the SIX1 and the SIX3/SIX5 locus. By comparing two different promoter deletions, we found that SIX1 expression in planta requires a $41 \mathrm{bp}$ region that includes one of the conserved TCGGCA elements that we found to be enriched in the SIX gene promoters (Figure 5). In contrast, SIX3 and SIX5 are not expressed from the two shorter promoter deletion strains, but expression of both genes is restored in the strain with the longest promoter deletion. The longest deletion additionally includes one of the TCGGCA elements (Figure 6A), which in this instance may mediate the action of a transcriptional repressor. The association of this element with upstream regions of effector genes is statistically significant (see Methods and Additional file 3 for details). Also, a perfect match to the extended motif (AAGTCGGCAGT) is present in the upstream regions of three genes encoding enzymes that we found in our analysis of the xylem sap proteome: FOXG_11769 on chromosome 10, encoding a glycosyl hydrolase and the closely related FOXG_14234 on the pathogenicity chromosome and FOXG_17180 on an unpositioned scaffold, encoding a peroxidasecatalases. Nevertheless, the function of this putative regulatory sequence remains to be established.

Interestingly, SIX1 as well as SIX3 and SIX5 were weakly expressed in all promoter deletion strains in vitro, but not in planta. In absence of a plant host, SIX gene expression is usually very low [26], while it is strongly induced upon plant infection [53]. SIX genes are only needed during plant infection; therefore the fungus might actively suppress $S I X$ gene expression in the absence of a plant host. One way of suppressing gene expression is by modification of chromatin to a repressive, closed state. Repressive chromatin structures often involve histone modifications such as H3K9 methylation [54]. One origin of such repressive chromatin structures 
is TE silencing, often guided by small RNAs transcribed from the TE [55]. In plants of the Solanaceae family, MITEs proximal to $R$ gene loci were shown to produce small RNAs that are recruiting the histone methylation machinery for TE silencing resulting in the formation of closed chromatin [56]. TE silencing of the MITEs surrounding the SIX genes might likewise create a repressive chromatin environment, which may serve as a first layer of SIX gene regulation. Upon stress, such as during plant infection, TEs might be derepressed as shown for the oomycete pathogen Phythophthora ramorum [57], thus creating an open chromatin structure. Binding of transcriptional activators or repressors would be possible in an open chromatin state and provide the basis for a second layer of regulation of SIX gene expression.

\section{Identification of novel effector candidates}

We identified eight novel (candidate) effector genes based on the presence of a mimp in their promoters and/or the presence of their protein product in xylem sap of infected plants. Five of these genes (SIX8b, SIX9, SIX11, SIX13, SIX14) were identified by the in silico search and validated by the analysis of xylem sap from infected tomato plants. Like the previously identified SIX1 and SIX7 genes, SIX10 escaped the in silico identification due to a sequencing gap close to its promoter. SIX12 encodes an unusual effector lacking a recognizable $\mathrm{N}$-terminal signal peptide for secretion via the classic Endoplasmatic-Reticulum/Golgi route. Nevertheless, the Six12 protein is present in the xylem sap of infected tomato plants and therefore might be secreted via an unconventional protein secretion route [58]. SIX13 encodes the only effector known so far that is located on a LS chromosome other than the pathogenicity chromosome. We also identified FoAVE1 as a gene harboring a mimp in its promoter, but we did not detect the FoAve1 protein in the xylem sap nor detected FoAVE1 mRNA in infected plants (results not shown). Apparently, in the strains used here FoAVE1 is not expressed during infection, although it was shown to be able to elicit $V e 1$-mediated resistance in a heterologous system [59]. FoAve1 might be part of a silent effector reservoir together with the other three genes that encode small, secreted proteins and harbor a mimp in their promoters, but are not expressed during infection.

Some of the genes we identified here have been subject to gene or segmental duplications. ORX1 is present in two similar but not identical copies on the pathogenicity chromosome (FOXG_14258, FOXG_14236; Additional file 1). Two other genes, FOXG_17460 on the pathogenicity chromosome and FOXG_17130 on chromosome 6, both encode a metalloprotease. Apart from a missing 3' end of FOXG_17460 due to a sequencing gap, the two genes and their promoters are identical, indicating a recent duplication event. SIX13 is also duplicated. In both cases the duplicated gene copies do not harbor a mimp in their promoter. SIX8b is present in four identical copies due to an intra- and interchromosomal segmental duplication within and between chromosome 3 and 6 [1]. This duplicated chromosomal segment corresponds to another small chromosome that can be transferred horizontally from the strain Fol007 [1]. Progeny strains possessing both the pathogenicity chromosome and the other small chromosome are more aggressive towards tomato than progeny strains with only the pathogenicity chromosome. At present, we do not know which gene(s) on the small chromosome (corresponding to sc18 in the Fol4287 genome) contributes to pathogenicity towards tomato - Six8b was not found in the xylem sap proteome.

In summary, mimps are associated with the promoters of all small in planta secreted proteins, as well as several enzymes. Our strategy for in silico detection of effector genes in F. oxysporum is limited by three factors: 1) imperfect conservation of the IRs of a mimp, 2) sequencing gaps in the genome assembly and 3) absence of a canonical N-terminal signal peptide for secretion. The impact of first two factors may be alleviated by more advanced methods for mimp detection and genome assembly. The third factor, absence of a canonical signal peptide, can be either due to secretion via an unconventional route or to a failure of SignalP to predict a signal peptide, as was the case for SIX5 or SIX8. In the latter case, incorporation of gene structure (intron/exon) predictions or transcript sequences will be helpful. Overall, our approach presents a powerful tool to predict novel effectors and other virulence factors in F. oxysporum.

\section{Conclusions}

Class II TEs are much less evenly distributed over the Fol pathogenicity chromosome than class I TEs. Effector genes reside as single genes or mini-gene clusters within class II TE-enriched chromosomal subregions. Two MITEs are closely associated with effector genes. A (partial) mimp is always present in effector gene promoter regions and a mFot5 is frequently present downstream of the effector gene mini-clusters. We could exclude a direct involvement of the mimp in effector gene expression by making promoter deletion strains for two effector gene loci followed by gene expression analysis and tomato pathogenicity assays. Overall, the unique association of effector genes and mimps allowed us to develop a method to successfully predict candidate effector genes. For most of these genes, the corresponding protein was found by mass spectrometry in the xylem sap of tomato during Fol infection. Our method can easily 
be extended to predict novel effector genes in Fo strains with different host specificities.

\section{Methods}

\section{Plant lines and fungal strains}

The following tomato (Solanum lycopersicum) lines were used ( $\mathrm{Fol}$ resistance genes between brackets): 90E402F (I-1) [60,61]; 90E341F (I-2) [62] and E779 (I-3) [60], C32 (no I gene) [63]. The following Fol strains were used: Fol007 (race 2), Fol4287 (race 2), Fol004 (race1), Fol4287SIX1p1189, Fol4287SIX1p1230, Fol4287SIXc3p539, Fol4287SIX3p807, Fol4287SIX3p859, Fol42874sge1, Fol007هsix1. See Rep et al. [45] for a more detailed description of the wild type Fol strains.

\section{Identification and annotation of TEs}

Repetitive DNA elements were identified by performing a self-BLASTN against the Fol4287 genome sequence, then using a custom PERL script (Amyott S.G. et al,,. manuscript in preparation), which identifies multi-copy sequences and sorts these repeated sequences into non-redundant families. Additional TEs with terminal inverted repeats were identified by search the Fol4287 genome for inverted repeats of at least $19 \mathrm{bp}$ encompassing at most $5 \mathrm{~kb}$ of sequence. Blast was used to find all instances (full length or partial) in the Fol4287 genome. Additional file 5 contains prototypes for all newly identified TEs.

\section{Promoter deletion constructs}

The promoter deletion constructs for the SIX1 and SIX3/SIX5 promoters were made by PCR amplification sequences of the sequences flanking the part of the promoter that was to be deleted for homologous recombination, and their insertion in front of and behind the hygromycin resistance gene in the vector pRW2h (see below). For SIX1: for both deletion constructs a $829 \mathrm{bp}$ upstream fragment was cloned into pRW2h [64] between the PacI and Acc65I sites and a1093 bp and 1052 bp downstream fragment, for the SIX1p1189 construct and the SIX1p1230 construct respectively, were cloned into pRW2h between at the XbaI site. For SIX3: a 1001 bp upstream fragment was cloned into pRW2h between the PacI and Acc651 sites and a 1332 bp (SIX3p539), a 1064 bp (SIX3p807) and a 1012 bp (SIX3p807) downstream fragment was cloned into the XbaI site of pRW2h. Transformation of these constructs to Fol4287 was done with Agrobacterium as described earlier [65].

\section{Tomato disease assay}

Ten days old tomato seedlings were inoculated with a fungal spore suspension and disease was scored after three weeks as described earlier [49]. The outcome of the disease assays was quantified in two ways: 1 ) average plant weight above the cotyledons and 2) phenotype scoring according to a disease index ranging from zero (no disease) to four (heavily diseased or dead) [49].

\section{Fol gene expression analysis}

For in vitro expression analysis, Fol mycelium was harvested after three days growth at $25^{\circ} \mathrm{C}$ and $175 \mathrm{rpm}$ in minimal growth medium (3\% sucrose, 1\% KNO3 and $0.17 \%$ yeast nitrogen base without amino acids and ammonia). For in planta expression analysis, ten days old tomato seedlings were inoculated with fungal spores suspensions as described above and roots were sampled eight or nine days after inoculation. From the collected material, RNA was isolated using TRIzol reagent (Gibco) followed by phenol-chloroform extraction. The isolated RNA was used to make cDNA using Promega Rnasin (ribonuclease inhibitor) and Gibco Superscript II RNaseH Reverse transcriptase according to the manufacturer's instructions. Primers used for RT-PCR analysis are listed in Additional file 6.

\section{Identification of novel effector candidates}

Based on published sequences of prototypes of mimp1-4 as well as mimps present in promoters of SIX1-7, a consensus mimp 3' IR was defined as 'TT[TA] TTGCNNCCCACTG'. A PERL script was used to find instances of this pattern in the genome sequence of Fol4287, downloaded from the broad website (http:// www.broadinstitute.org/annotation/genome/

fusarium_group/MultiHome.html). For 150 of the 158 matches to this pattern, the next dinucleotide was 'TA', which is the required target site for mimps and Impalas.

For each mimp IR match, all open reading frames (ORFs) starting with an ATG and of at least 25 codons within $2000 \mathrm{bp}$ downstream of the IR were selected. The ORFs were translated and the translation products submitted to signal peptide prediction by SignalP (http:// www.cbs.dtu.dk/services/SignalP/). If positive, the instance was recorded (mimp IR sequence, translation product of ORF and their positions in the Fol4287 contig). The sequence surrounding this instance was retrieved and manually inspected to define the full ORF of the candidate effector gene.

\section{In silico promoter analysis}

To find potential regulatory elements in promoters of effector genes, we first identified enriched k-mers in the concatenated upstream regions of SIX1, SIX2, SIX3, SIX5, SIX6 and SIX7, using Compseq (http://emboss.bioinformatics.nl/cgi-bin/emboss/compseq). As upstream regions we used here the sequences between the upstream mimp and the ATG, to avoid identification of sequences within mimps (especially the conserved inverted repeats). We looked for enriched 6mers, 7 mers, 
8 mers and 9 mers in both strands. Among the most frequent 6 mers and 7 mers, we found two classes: (1) A diversity of AT-sequences and (2) a small set of overlapping sequences that were present in one or more instances in all - or all but one - upstream regions. The most frequent sequence elements of the second class were the 6mers TCGGCA (16), GGCAGT (14), CGGCAG (11) and GCAGTT (11) and the 7mers GGCAGTT (11), TCGGCAG (9) and CGGCAGT (7). The overlap of these 6 mers and 7 mers is the 9 mer TCGGCAGTT. This is also the most frequent 9mer, which occurs 6 times in the upstreams regions, namely in those of SIX1 (2X), SIX3 (1X) and SIX5 (3X). Except two palindromic AT-rich sequences (TTTTAAAA and TATATATA), the most frequent 8 mers matched this 9mer: TCGGCAGT (6), CGGCAGTT (7), or extend it: GGCAGTTA (6). Two other frequent 8mers extend the sequence on the other end: AAGTCGGC (4) and AGTCGGCA (4). Additional overlapping, enriched 9mers (each occuring 3 times) further extend the combined sequence to the consensus AAGTCGGCAGTT[AG]A.

To assess the significance of the occurrence of this motif in the upstream regions of effector genes, we analysed the 17708 upstream regions of Fol4287 genes, defined as 1000 bp upstream of the predicted translational start codon. This analysis is summarized in Additional file 3. Briefly, we calculated the probability that the frequency with which the two most frequent (and overlapping) 6mers, TCGGCA and GGCAGT, and to the most frequent 7mer, GGCAGTT (a one base extension to the second $6 \mathrm{mer}$ ), occur at least once or twice in the upstream regions of effector genes is by chance association. We did this both for the original set of effector genes used to find the pattern (SIX1-3 and SIX57 ), and for the entire set of identified effector genes (including SIX8b). All p values were lower than 0.05. The weakest association was between at least one TCGGCA element and the original set $(\mathrm{p}=0.024)$ and between at least one GGCAGT and the entire set $(\mathrm{p}=0.015)$. Association with at least twice occurences were more significant in all cases. Association with the entire set was slightly more significant for the TCGGCA element (at least once or at least twice) and for at least twice occurences of the GGCAGT element. The other associations were weaker with the entire set of effector genes.

\section{Xylem sap collection, mass spectrometry and label free} quantitative proteomics

Fol007 was used for tomato inoculation. Four-week-old tomato plants C32 were inoculated, after removing part of the root system, with a Fol spore suspension $\left(5 \times 10^{6}\right.$ spores $\mathrm{mL}^{-1}$ ) or with water as a negative control, and potted. Fourteen days post inoculation (dpi), xylem sap was collected as described [66,67]. Briefly, stems were cut below the second true leaf and the plant was placed in a horizontal position. Then, for minimal $6 \mathrm{~h}$ sap bleeding from the cut surface was collected in tubes placed on ice. The collected xylem sap was stored at $-20^{\circ} \mathrm{C}$.

For label-free protein quantification 25 plants per inoculum were inoculated with Fol007 or water. Xylem sap was isolated as described above from four independent biological replicates. A fraction of the sap was used for immunoblotting, the remainder was concentrated with a Centricon plus-70 (Millipore) unit to a final volume of 200-300 $\mu \mathrm{l}$. The protein concentration was determined with the bicinchoninic acid method (Sigma). After trichloroacetic acid/aceton precipitation protein isolated from inoculated plants with water or Fol007 was dissolved in sample buffer at equal concentration (1.5 $\mu \mathrm{g} / \mu \mathrm{l})$ and $30 \mu \mathrm{l}$ per sample was loaded on the SDSPAGE. SDS-PAGE was performed with Hoefer Mighty Small SE250 minigel equipment (Amersham Biosciences, $\mathrm{AB}$, Uppsala). After a short run, the Coommassie PageBlue $^{\mathrm{Tm}}$ (Fermentas) was used to visualize the proteins in the SDS-PAGE. For each xylem sap sample one gel slice containing all proteins was cut from the Coomassie-stained gel. In-gel digestion was performed as described by Rep et al. [67]. The peptides obtained after this digestion were analyzed by nanoLC-MS/MS as described by $\mathrm{Lu}$, et al [68]. Raw data from the LTQOrbitrap were analyzed with MaxQuant software $[69,70]$ to identify the proteins and allow label-free relative quantification. MaxQuant 1.1.36 settings were used according to the description by Peng, et al [71]. The Fol protein database used for the analysis was obtained from Fusarium Comparative Genome website (http://www. broadinstitute.org/annotation/genome/fusarium_group/ MultiHome.html) and supplemented by adding the sequences of known Six proteins that are not annotated in the public database. A "contaminant" database was used that contains proteins such as trypsin and human keratins [71]. Bioinformatics analysis of the MaxQuant workflow and the statistical analysis of the abundances of the identified proteins were performed using Perseus (available at www.MaxQuant.org) [70]. Only proteins identified with at least two peptides, of which one should be unique, were kept.

\section{Additional files}

Additional file 1: Detailed annotation of the Fol4287 pathogenicity chromosome.

Additional file 2: A putative secondary metabolite gene cluster of $\mathrm{Fol}$ is expressed during tomato infection. Roots of ten days old susceptible (without resistance genes) tomato seedlings were inoculated 
with conidiospores of Fol004. Roots were harvested 8 dpi (days post inoculation). From the collected roots RNA was extracted and (RT-) PCR was performed to detect transcripts of the indicated genes. Numbers represent FOXG numbers of the Fol4287 reference genome. Marker sizes are indicated on the right. C: CDNA, G: genomic DNA.

Additional file 3: Significance of the association between the TCGGCA element and upstream regions of effector genes.

Additional file 4: Complex repeat structure in SIX8, SIX8b and SIX14 upstream regions. The most upstream sequence shared between the SIX8 and SIX86 loci (dark grey, blue and green highlighted) is more similar between $S I X 8$ and $S I X 8 b$ loci than the coding sequences and the immediate upstream sequences (light grey). The SIX8b upstream region is the most complex. Compared to that of SIX8, there are: (a) a mimp4 insertion, (b) a Han insertion, (c) an inversion and duplication (indicated with < signs), (d) a mimp1 insertion, (e) a partial mimp3 and (f) an extra sequence that includes an mFot5. A total of 9 mimp-related inverted repeats are present, of which two are interrupted by a TE. Part of the SIX14 upstream region is almost identical to a part of the SIX8b upstream region (green/blue highlighted including the mimp4) - except that the Han insertion is missing in the SIX14 locus. In both cases, a mimp1 is present immediately downstream of this region but, though similar in sequence, these mimp1 insertions appear to be independent. Blue capital letters: effector ORF (introns in lower case); Green capital letters: mimp; Dark red capital letters: mFot5; Orange capital letters: Han; Gray highlight: shared between SIX8 and SIX8b loci only; Light gray highlight: similarity between $S I X 8$ and $S I X 86$ upstream (leader/promoter) sequences; Blue highlight: mimp-like inverted repeat sequence, present one or more times in SIX8, SIX8b and SIX14 loci (numbers of likely orthologous sequences correspond between the three loci - note that mimp-IR1 does not conform to the consensus sequence for mimp inverted repeats); Green and dark green highlight: sequences present one or more times in SIX8, SIX8b and SIXI4 loci; Yellow highlight: TGCCGA motif; Bold: target site duplications associated with TE insertions.

Additional file 5: Newly identified TEs of Fol.

Additional file 6: Primers used in this study.

\section{Competing interests}

The authors declare that they have no competing interests.

\section{Authors' contributions}

SMS and MR designed experiments, carried out the studies and analysis and wrote the manuscript. PMH helped generate the expression data and tomato pathogenicity test. IS made the promoter deletion constructs. LM and SB generated the MS analysis of the tomato xylem sap proteome. SA helped identifying transposable elements in the Fo/4287 genome. BC generated expression data of the secondary metabolite cluster. All authors read and approved the final manuscript.

\section{Acknowledgements}

We thank Chiara Bertoldo for help with the RNA isolation and Ben Cornelissen for helpful comments on the manuscript. SMS and MR are funded by a NWO Vici Grant. IS was supported by a EU Erasmus scholarship. All proteomic LC MS/MS measurements were done at Biqualys Wageningen (www.biqualys.nl) and funded by the Centre for BioSystems Genomics (CBSG).

\section{Author details}

'Plant Pathology, Swammerdam Institute for Life Sciences, University of Amsterdam, Science Park 904, 1098 XH, Amsterdam, the Netherlands. ${ }^{2}$ Department of Plant Pathology, University of Kentucky, 201F Plant Science Building, 1405 Veterans Drive, Lexington, KY 40546-0312, USA. ${ }^{3}$ Laboratory for Biochemistry, Wageningen University, Dreijenlaan 3, Wageningen 6703HA, the Netherlands. ${ }^{4}$ Current address: Fachgebiet Medizinische Biotechnologie, Institut für Biotechnologie, Technische Universität Berlin, Gustav-Meyer-Allee 25, Berlin, Germany.

Received: 1 November 2012 Accepted: 11 February 2013 Published: 22 February 2013

\section{References}

1. Ma L-J, van der Does HC, Borkovich KA, Coleman JJ, Daboussi M-J, Di Pietro A, Dufresne M, Freitag M, Grabherr M, Henrissat B, et al: Comparative genomics reveals mobile pathogenicity chromosomes in Fusarium. Nature 2010, 464(7287):367-373.

2. Rep M, Kistler HC: The genomic organization of plant pathogenicity in Fusarium species. Curr Opin Plant Biol 2010, 13(4):420-426.

3. Recorbet G, Steinberg C, Olivain C, Edel V, Trouvelot S, Dumas-Gaudot E, Gianinazzi S, Alabouvette C: Wanted: pathogenesis-related marker molecules for Fusarium oxysporum. New Phytol 2003, 159(1):73-92

4. Lievens B, Rep M, Thomma BP: Recent developments in the molecular discrimination of formae speciales of Fusarium oxysporum. Pest Manag Sci 2008, 64(8):781-788.

5. O'Donnell K, Cigelnik E, Casper HH: Molecular phylogenetic, morphological, and mycotoxin data support reidentification of the Quorn mycoprotein fungus as Fusarium venenatum. Fungal Genet Biol 1998, 23(1):57-67.

6. Baayen RP, O'Donnell K, Bonants PJ, Cigelnik E, Kroon LP, Roebroeck EJ, Waalwijk C: Gene genealogies and AFLP analyses in the Fusarium oxysporum complex identify monophyletic and nonmonophyletic formae speciales causing wilt and Rot disease. Phytopathology 2000, 90(8):891-900.

7. De Wit PJGM, Mehrabi R, Van Den Burg HA, Stergiopoulos I: Fungal effector proteins: past, present and future. Mol Plant Pathol 2009, 10(6):735-747.

8. Rep M: Small proteins of plant-pathogenic fungi secreted during host colonization. FEMS Microbiol Lett 2005, 253(1):19-27.

9. Michielse CB, van Wijk R, Reijnen L, Cornelissen BJC, Rep M: Insight into the molecular requirements for pathogenicity of Fusarium oxysporum $\mathrm{f}$. sp. lycopersici through large-scale insertional mutagenesis. Genome Biol 2009, 10(1):R4

10. Raffaele S, Win J, Cano LM, Kamoun S: Analyses of genome architecture and gene expression reveal novel candidate virulence factors in the secretome of Phytophthora infestans. BMC Genom 2010, 11(1):637.

11. Rouxel T, Grandaubert J, Hane JK, Hoede C, van de Wouw AP, Couloux A, Dominguez V, Anthouard V, Bally P, Bourras S, et al: Effector diversification within compartments of the Leptosphaeria maculans genome affected by repeat-induced point mutations. Nat Commun 2011, 2:202.

12. Chuma I, Isobe C, Hotta Y, Ibaragi K, Futamata N, Kusaba M, Yoshida K, Terauchi R, Fujita Y, Nakayashiki H, et al: Multiple translocation of the AVRpita effector gene among chromosomes of the rice blast fungus Magnaporthe oryzae and related species. PLoS Pathog 2011, 7(7):e1002147.

13. Finnegan DJ: Eukaryotic transposable elements and genome evolution. Trends Genet 1989, 5(4):103-107.

14. Wicker T, Sabot F, Hua-Van A, Bennetzen UL, Capy P, Chalhoub B, Flavell A, Leroy $P$, Morgante M, Panaud O, et al: A unified classification system for eukaryotic transposable elements. Nat Rev Genet 2007, 8(12):973-982.

15. Feschotte $C$, Pritham EJ: DNA transposons and the evolution of eukaryotic genomes. Annu Rev Genet 2007, 41(1):331-368.

16. Kang S, Lebrun MH, Farrall L, Valent B: Gain of virulence caused by insertion of a Pot3 transposon in a Magnaporthe grisea avirulence gene. Mol Plant Microbe Interact 2001, 14(5):671-674.

17. Inami K, Yoshioka-Akiyama C, Morita Y, Yamasaki M, Teraoka T, Arie T: A genetic mechanism for emergence of races in Fusarium oxysporum $\mathrm{f}$. sp. Lycopersici: inactivation of avirulence gene AVR1 by transposon insertion. PLoS One 2012, 7(8):e44101.

18. Houterman PM, Ma L, van Ooijen G, de Vroomen MJ, Cornelissen BJC, Takken FLW, Rep M: The effector protein Avr2 of the xylem-colonizing fungusFusarium oxysporumactivates the tomato resistance protein I-2 intracellularly. Plant J 2009, 58(6):970-978.

19. Houterman PM, Speijer D, Dekker HL, De Koster CG, Cornelissen BJC, Rep M: The mixed xylem sap proteome of Fusarium oxysporum-infected tomato plants. Mol Plant Pathol 2007, 8(2):215-221.

20. van der Does HC, Lievens B, Claes L, Houterman PM, Cornelissen BJ, Rep M: The presence of a virulence locus discriminates Fusarium oxysporum isolates causing tomato wilt from other isolates. Environ Microbiol 2008, 10(6):1475-1485.

21. Lee T, Han Y-K, Kim K-H, Yun S-H, Lee Y-W: Tri13 And Tri7 determine Deoxynivalenol- and Nivalenol-producing chemotypes of Gibberella zeae. Appl Environ Microbiol 2002, 68(5):2148-2154.

22. Mehrabi R, Bahkali AH, Abd-Elsalam KA, Moslem M, Ben M'Barek S, Gohari AM, Jashni MK, Stergiopoulos I, Kema GHJ, de Wit PJGM: 
Horizontal gene and chromosome transfer in plant pathogenic fungi affecting host range. FEMS Microbiol Rev 2011, 35(3):542-554

23. Roca MG, Arlt J, Jeffree CE, Read ND: Cell biology of conidial anastomosis tubes in Neurospora crassa. Eukaryot Cell 2005, 4(5):911-919.

24. Saupe SJ, Glass NL: Allelic specificity at the het-c heterokaryon incompatibility locus of Neurospora crassa is determined by a highly variable domain. Genetics 1997, 146(4):1299-1309.

25. Paoletti M, Saupe SJ, Clavé C: Genesis of a fungal non-self recognition repertoire. PLOS One 2007, 2(3):e283.

26. Michielse $C B$, van Wijk R, Reijnen $L$, Manders EM, Boas S, Olivain $C$, Alabouvette C, Rep M: The nuclear protein Sge1 of Fusarium oxysporum is required for parasitic growth. PLoS Pathog 2009, 5 (10):e1000637.

27. Ramos B, Alves-Santos FM, García-Sánchez MA, Martín-Rodrigues N, Eslava AP, Díaz-Mínguez JM: The gene coding for a new transcription factor ( $\mathrm{ft}$ 1) of Fusarium oxysporum is only expressed during infection of common bean. Fungal Genet Biol 2007, 44(9):864-876.

28. Dantzer F, Schreiber V, Niedergang C, Trucco C, Flatter E, Rubia GDL, Oliver J, Rolli V, Ménissier-de Murcia J, de Murcia G: Involvement of poly (ADP-ribose) polymerase in base excision repair. Biochimie 1999, 81(1,Äi2):69-75.

29. Kline SL, Cheeseman IM, Hori T, Fukagawa T, Desai A: The human Mis12 complex is required for kinetochore assembly and proper chromosome segregation. J Cell Biol 2006, 173(1):9-17.

30. Goshima G, Kiyomitsu T, Yoda K, Yanagida M: Human centromere chromatin protein $\mathrm{hMis} 12$, essential for equal segregation, is independent of CENP-A loading pathway. J Cell Biol 2003, 160(1):25-39.

31. Jones DO, Cowell IG, Singh PB: Mammalian chromodomain proteins: their role in genome organisation and expression. Bioessays 2000, 22(2):124-137.

32. Spanu PD, Abbott JC, Amselem J, Burgis TA, Soanes DM, Stuber K, Loren van Themaat EV, Brown JKM, Butcher SA, Gurr SJ, et al: Genome expansion and gene loss in powdery mildew fungi reveal tradeoffs in extreme parasitism. Science 2010, 330(6010):1543-1546.

33. Walbot V: Maize genome in motion. Genome Biol 2008, 9(4):303.

34. Wessler SR: Transposable elements and the evolution of eukaryotic genomes. Proc Natl Acad Sci 2006, 103(47):17600-17601.

35. Ma J, Devos KM, Bennetzen JL: Analyses of LTR-retrotransposon structures reveal recent and rapid genomic DNA loss in rice. Genome Res 2004, 14 (5):860-869.

36. Shiflett A, Enkerli J, Covert S: Nht2, a copia LTR retrotransposon from a conditionally dispensable chromosome in Nectria haematococca. Curr Genet 2002, 41(2):99-106.

37. Mes JJ, Haring MA, Cornelissen BJ: Foxy: an active family of short interspersed nuclear elements from Fusarium oxysporum. Mol Gen Genet 2000, 263(2):271-280.

38. Teunissen HAS, Rep M, Houterman PM, Cornelissen BJC, Haring MA: Construction of a mitotic linkage map of Fusarium oxysporum based on Foxy \& AFLPs. Mol Genet Genomics 2003, 269(2):215-226.

39. Goodwin TJ, Butler MI, Poulter RT: Cryptons: a group of tyrosinerecombinase-encoding DNA transposons from pathogenic fungi. Microbiology 2003, 149(Pt 11):3099-3109.

40. Kojima K, Jurka J: Crypton transposons: identification of new diverse families and ancient domestication events. Mob DNA 2011, 2(1):12

41. Dufresne M, Lespinet O, Daboussi M-J, Hua-Van A: Genome-wide comparative analysis of pogo-like transposable elements in different Fusarium species. J Mol Evol 2011, 73(3-4):230-243.

42. Chalvet F: Hop, an active Mutator-like element in the genome of the fungus Fusarium oxysporum. Mol Biol Evol 2003, 20(8):1362-1375.

43. Gomez-Gomez E, Anaya N, Roncero MIG, Hera C: Folyt1, A New member of the hAT family, is active in the genome of the plant pathogen Fusarium oxysporum. Fungal Genet Biol 1999, 27(1):67-76.

44. Hua-Van A, Daviere JM, Kaper F, Langin T, Daboussi MJ: Genome organization in Fusarium oxysporum: clusters of class II transposons. Curr Genet 2000, 37(5):339-347.

45. Rep M, van der Does HC, Cornelissen BJC: Drifter, a novel, low copy hATlike transposon in Fusarium oxysporum is activated during starvation. Fungal Genet Biol 2005, 42(6):546-553.

46. Langin T, Capy P, Daboussi MJ: The transposable element Impala, a fungal member of the TC1-Mariner superfamily. Mol Gen Genet 1995, 246(1):19-28.
47. Hua-Van A, Hericourt F, Capy P, Daboussi MJ, Langin T: Three highly divergent subfamilies of the Impala transposable element coexist in the genome of the fungus Fusarium oxysporum. Mol Gen Genet 1998, 259(4):354-362.

48. Daboussi M-J, Capy P: Transposable elements in filamentous fungi. Annu Rev Microbiol 2003, 57(1):275-299.

49. Rep M, Van Der Does HC, Meijer M, Van Wijk R, Houterman PM, Dekker HL, De Koster CG, Cornelissen BJC: A small, cysteine-rich protein secreted by Fusarium oxysporum during colonization of xylem vessels is required for I-3-mediated resistance in tomato. Mol Microbiol 2004, 53(5):1373-1383.

50. Batada NN, Urrutia AO, Hurst LD: Chromatin remodelling is a major source of coexpression of linked genes in yeast. Trends Genet 2007, 23(10):480-484.

51. Bergemann M, Lespinet O, M'Barek SB, Daboussi M-J, Dufresne M: Genomewide analysis of the Fusarium oxysporum mimp family of MITEs and mobilization of both native and De novo created mimps. J Mol Evol 2008, 67(6):631-642

52. Dufresne M, Hua-Van A, Abd el Wahab H, M'Barek SB, Vasnier C, Teysset L, Kema GHJ, Daboussi MJ: Transposition of a fungal miniature invertedrepeat transposable element through the action of a Tc1-like transposase. Genetics 2006, 175(1):441-452.

53. van der Does HC, Duyvesteijn RG, Goltstein PM, van Schie CC, Manders EM, Cornelissen BJ, Rep M: Expression of effector gene SIX1 of Fusarium oxysporum requires living plant cells. Fungal Genet Biol 2008, 45(9):1257-1264

54. Li B, Carey M, Workman JL: The role of chromatin during transcription. Cell 2007, 128(4):707-719.

55. Beisel C, Paro R: Silencing chromatin: comparing modes and mechanisms. Nat Rev Genet 2011, 12(2):123-135.

56. Kuang H, Padmanabhan C, Li F, Kamei A, Bhaskar PB, Ouyang S, Jiang J, Buell $C R$, Baker B: Identification of miniature inverted-repeat transposable elements (MITEs) and biogenesis of their siRNAs in the Solanaceae: new functional implications for MITEs. Genome Res 2009, 19(1):42-56.

57. Kasuga T, Kozanitas M, Bui M, Hueberli D, Rizzo DM, Garbelotto M: Phenotypic diversification is associated with host-induced transposon derepression in the sudden Oak death pathogen Phytophthora ramorum. PLoS One 2012, 7(4):e34728.

58. Nickel W: Pathways of unconventional protein secretion. Curr Opin Biotechnol 2010, 21(5):621-626.

59. de Jonge R, Peter van Esse H, Maruthachalam K, Bolton MD, Santhanam P, Saber MK, Zhang Z, Usami T, Lievens B, Subbarao KV, et al: Tomato immune receptor Ve1 recognizes effector of multiple fungal pathogens uncovered by genome and RNA sequencing. Proc Natl Acad Sci 2012, 109 (13):5110-5115.

60. Scott JW, Jones JP: Monogenic resistance in tomato to Fusarium oxysporum f. sp. lycopersici race 3. Euphytica 1989, 40(1):49-53.

61. Mes JJ, Wit R, Testerink CS, de Groot F, Haring MA, Cornelissen BJC: Loss of avirulence and reduced pathogenicity of a gamma-irradiated mutant of Fusarium oxysporum f. sp. Lycopersici. Phytopathology 1999, 89(12):1131-1137.

62. Stall RE, Walter JM: Selection and inheritance of resistance in tomato to isolates of races 1 and 2 of the Fusarium wilt organism. Phytopathology 1965, 55:1213-1215.

63. Kroon BAM, Elgersma DM: Interactions between race 2 of Fusarium oxysporum f. sp. Lycopersici and near-Isogenic resistant and susceptible lines of intact plants or callus of tomato 58731 F71 137. J Phytopathol 1993, 137(1):1-9

64. Houterman PM, Cornelissen BJC, Rep M: Suppression of plant resistance gene-based immunity by a fungal effector. PLoS Pathog 2008, 4(5):e1000061.

65. Takken FL, Van Wijk R, Michielse CB, Houterman PM, Ram AF, Cornelissen BJ: A one-step method to convert vectors into binary vectors suited for Agrobacterium-mediated transformation. Curr Genet 2004, 45(4):242-248.

66. Krasikov V, Dekker HL, Rep M, Takken FL: The tomato xylem sap protein XSP10 is required for full susceptibility to Fusarium wilt disease. J Exp Bot 2011, 62:963-973.

67. Rep M: Mass spectrometric identification of isoforms of PR proteins in xylem sap of fungus-infected tomato. Plant Physiol 2002, 130(2):904-917.

68. Lu S-W, Yun S-H, Lee T, Turgeon BG: Altering sexual reproductive mode by interspecific exchange of MAT loci. Fungal Genet Biol 2011, 48(7):714-724 
69. Cox J, Mann M: MaxQuant enables high peptide identification rates, individualized p.p.b.-range mass accuracies and proteome-wide protein quantification. Nat Biotechnol 2008, 26:1367-1372.

70. Hubner NC, Bird AW, Cox J, Splettstoesser B, Bandilla P, Poser I, Hyman A, Mann M: Quantitative proteomics combined with BAC TransgeneOmics reveals in vivo protein interactions. J Cell Biol 2010, 189:739-754.

71. Peng K, van Lent JW, Boeren S, Fang M, Theilmann DA, Erlandson MA, Vlak $J M$, van Oers MM: Characterization of novel components of the baculovirus per os infectivity factor (PIF) complex. J Virol 2012. 86(9):4981-4988

doi:10.1186/1471-2164-14-119

Cite this article as: Schmidt et al:: MITEs in the promoters of effector genes allow prediction of novel virulence genes in Fusarium oxysporum. BMC Genomics 2013 14:119.

\section{Submit your next manuscript to BioMed Central and take full advantage of:}

- Convenient online submission

- Thorough peer review

- No space constraints or color figure charges

- Immediate publication on acceptance

- Inclusion in PubMed, CAS, Scopus and Google Scholar

- Research which is freely available for redistribution 